\title{
多糖化学合成研究进展
}

\author{
吴勇叶新山* \\ (天然药物及仿生药物国家重点实验室 北京大学药学院 北京 100191)
}

\begin{abstract}
摘要 多糖是一类结构复杂的生物大分子, 广泛分布于微生物、植物、动物等生物体中, 参与众多重要的生命过程. 然 而, 多糖研究一直受限于难以获得足量结构均一的多糖物质, 这使得多糖的结构和功能研究及其相关的应用研究进展 缓慢. 近几十年来, 随着糖合成技术的进步, 糖的化学合成效率不断提高, 人们开始关注复杂多糖的化学合成, 并取得 了一些进展. 本文主要对多糖化学合成所涉及的糖基化方法、糖组装策略和代表性的合成工作进行综述, 并对研究前 景进行展望.
\end{abstract}

关键词 多糖; 化学合成; 糖基化方法; 合成策略

\section{Recent Advances in Chemical Synthesis of Polysaccharides}

\author{
Wu, Yong Ye, Xin-Shan* \\ (State Key Laboratory of Natural and Biomimetic Drugs, School of Pharmaceutical Sciences, Peking University, Beijing \\ 100191)
}

Abstract Polysaccharides are a class of bio-macromolecules with highly complex structures that are widely found in living organisms such as microorganisms, plants and animals. Polysaccharides serve not only as structural components and energy sources of cells, but also as important signaling molecules which are involved in many key biological processes. Studies on polysaccharide-mediated biological processes require access to structurally defined molecules, which approach the size and complexity of those found in nature, but naturally-occurring polysaccharides usually exist in microheterogeneous forms, making it difficult or even impossible to isolate pure polysaccharides from natural sources in most cases. Chemical synthesis represents a reliable solution to this problem, which can provide polysaccharide samples with defined chemical structures for functional studies and even a library of analogs of natural glycans for structure-activity relationship investigations. But unlike oligonucleotides and peptides, which can already be obtained by automated synthesizers in a very short of time, the chemical synthesis of glycans remains a great challenge for synthetic chemists. The major challenge for glycan synthesis lies in the need to handle both stereo- and regio-chemistry in the construction of each glycosyl linkage, and the extensive protecting-group manipulations as well as much intermediate separation make it a tedious and time-consuming process. Over the past decades, carbohydrate chemists have developed many glycosylation reactions. A series of strategies for glycan assembly have been also established. The advances in both synthetic methods and strategies have significantly increased the synthetic efficiency of carbohydrate molecules, and many great accomplishments in the field of polysaccharide synthesis have been witnessed in recent decades. Some representative methods and strategies, and their successful applications in the chemical synthesis of complex polysaccharides are summarized in this review.

Keywords polysaccharide; chemical synthesis; glycosylation method; synthetic strategy
\end{abstract}

\section{1 引言}

糖类化合物(Carbohydrates)是自然界中分布最为广 泛的一类生物分子, 与核酸、蛋白质和脂类共同构成了 细胞的基本组成. 长久以来, 糖类主要被视为生物体的 结构物质(如纤维素)和能量物质(如淀粉和糖原). 然而 随着糖生物学研究的不断深入, 人们越来越意识到糖类 是一类重要的信息分子, 广泛参与病原体感染、免疫应 答、细胞信号转导、细胞分裂和分化、炎症反应、精卵 识别和肿瘤转移等生命过程 ${ }^{[1,2]}$. 另一方面, 糖类也是重 要的药用分子, 比如被广泛应用于疾病治疗和预防的抗
凝药肝素、氨基糖苷类抗生素以及细菌多糖结合疫苗 等 ${ }^{[3,4]}$. 此外, 糖类还常作为 “手性池” 起始原料, 用于 合成具有重要生物活性及应用价值的天然产物或医药 中间体 ${ }^{[5]}$.

聚糖的基本结构单元为单糖，单糖与单糖之间以糖 苷键相连, 形成线性或者分支的聚糖结构. 按照所含单 糖单元数目的多少, 聚糖又可分为臭聚糖(寡糖)和多聚 糖(多糖). 寡糖和多糖之间并没有明显的界限, 在 20 世 纪 70 年代,一般超过 10 个单糖单元组成的聚糖结构就 可以被称为多糖; 而随着糖合成技术的进步，合成的糖

\footnotetext{
* E-mail: xinshan@bjmu.edu.cn; Tel.: 010-82805736; Fax: 010-82802724

Received April 11, 2019; published June 6, 2019.

Project supported by the National Natural Science Foundation of China (No. 21738001).

项目受国家自然科学基金重点项目(No. 21738001)资助.
} 
越来越大, 目前一般可以将超过 20 个单糖单元所组成 的聚糖称为多糖.

天然存在的多糖(如糖胺聚糖、细菌荚膜多糖等)大 多结构复杂, 且存在微观不均一性, 这使得人们难以通 过分离纯化的方式得到足量纯净的多糖样品. 另外, 多 糖的生物合成没有固定的模板, 不由基因直接编码, 因 此也无法像核酸或者蛋白质那样通过 PCR 或者基因表 达的方式获取样品 ${ }^{[6]}$. 多糖样品的获得性问题成为限制 其生物学功能和构效关系研究的瓶颈.

化学合成是获取均一结构多糖的重要途径. 但由于 多糖的结构十分复杂, 且受限于糖合成的水平, 人们通 常选择短的糖片段进行合成. 在某些情况下, 合成短的 糖链就能够满足研究需要, 然而在分子尺寸依赖的生物 识别过程中 ${ }^{[7]}$, 短的糖链往往难以模拟天然多糖的复杂 结构. 例如 Takahashi 等 ${ }^{[8]}$ 发现 $\beta-1,3$ 连接的葡聚糖与受 体 Dectin-1 的结合力随糖链长度增加而增强, 但即便是 十七糖, 其结合力也比天然的多糖弱很多; 另外, Petitou 等 $^{[9]}$ 在研究合成的小分子肝素抑制凝血酶活性时发 现, 合成的十五糖(约 $5.5 \mathrm{kD}$ )比天然肝素(约 $15 \mathrm{kD}$ )的 $\mathrm{IC}_{50}$ 仍小一个数量级. 此外, 细菌的抗原性多糖成分(或 其蛋白结合物)往往能够刺激机体产生强的免疫反应, 但有时使用合成的短的糖链所引起的抗体滴度却很 低 ${ }^{[10]}$.

合成分子尺寸和复杂性与天然多糖相当的聚糖化 合物有助于从分子水平理解多糖的生物学功能及构效 关系. 近几十年来, 糖化学家们发展了大量新颖的糖基 化方法, 发展了高效的糖链组装策略, 并且开始运用这 些方法和策略进行复杂结构多糖的化学合成. 目前, 已 经有多篇综述性文章对糖基化方法和糖链组装策略进 行了详细的总结 ${ }^{[11]}$, 本文将择其代表性的方法和策略进 行简介, 重点对其在复杂多糖合成中的应用进行综述.

\section{2 聚糖化学合成方法}

\section{1 糖基化反应概述}

聚糖化学合成中最重要的反应是糖单元与糖单元 之间的偶联反应——糖基化反应(Glycosylation reaction). 通常来讲, 糖基化反应的底物包括两种: 其一为 端基带某种离去基团(Leaving group, LG)的糖基供体 (Donor), 其二为带有亲核反应基团的糖基受体 (Acceptor). 根据受体亲核反应基团的不同，糖基化反 应可以分为氧一糖基化反应、氮-糖基化反应和碳-糖基 化反应等, 相应的糖基化反应产物被称作氧苷、氮苷和 碳苷等.

以氧-糖基化为例，一般的反应过程如图 1 所示，供 体 1 被促进剂(Promoter) 活化后, 生成氧鎓离子 (Oxocarbenium ion)中间体 2. 当 C-2 位存在邻基参与基 团(Participating groups)时, 受体 $\left(\mathrm{R}^{1}-\mathrm{OH}\right)$ 从邻基参与基 团的反方向进攻端基碳，从而生成 1,2-反式糖甘 3 (1,2-trans glycoside). 常见的邻基参与基团主要为酯型 保护基，如乙酰基和苯甲酰基等.

而当 C-2 位不存在邻基参与基团时，则受体可以从 糖环的 $\alpha$ 和 $\beta$ 面进攻端基碳, 从而生成 1,2-反式和 1,2顺式糖苷(1,2-cis glycoside)混合物 4. 反式和顺式的比 例受很多因素影响，比如端基效应、离去基、保护基、 受体性质和反应条件(如活化方式、添加剂、溶剂、压 力、温度、浓度)等.

\section{2 常用的糖基化方法}

最早的糖基化反应可以追溯到 19 世纪末期. 1879 年, Michael 等 ${ }^{[12]}$ 首次报道了使用氯/溴代糖作为糖基供 体, 与亲核性较强的苯酚钾盐发生糖基化反应合成简单 酚苷的例子. 自那以后，人们在发展糖基化方法方面做 了大量工作, 并陆续报道了数十种新颖的糖基供体,

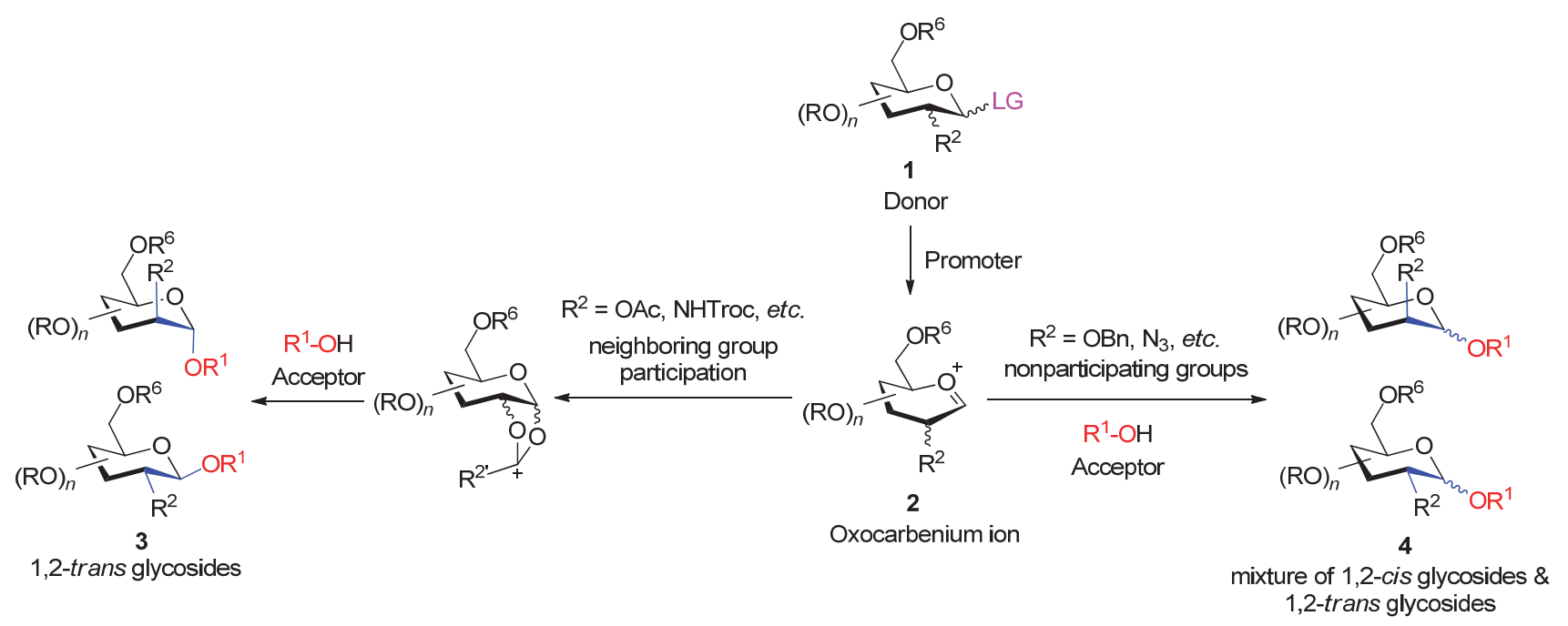

图 1 糖基化的一般反应过程

Figure 1 General mechanism of glycosylation reactions 
如卤代糖供体 ${ }^{[13]}$ 、半缩醛糖基供体 ${ }^{[14]}$ 、三氯乙酰亚胺酯 供体 ${ }^{[15]}$ 、硫苷供体 ${ }^{[16]}$ 、戊烯苷供体 ${ }^{[17]}$ 、糖烯供体 ${ }^{[18]}$ 、 糖基磷酸酯供体 ${ }^{[19]}$ 、邻炔基苯甲酸酯糖基供体 ${ }^{[20]}$ 等. 随 着这些糖基供体的出现, 还发展出各式各样与之匹配的 活化体系; 这些糖基化方法有各自的适用范围, 在聚糖 的化学合成中各显神通.

在众多的糖基供体中, 最常用的是卤代糖 ${ }^{[13]}$ 、糖基 三氯乙酰亚胺酯 ${ }^{[15]}$ 和硫苷 ${ }^{[16]}$.

\subsection{1 卤代糖作为糖基供体}

卤代糖(Glycosyl halide)是糖的端基为卤素取代的 一类糖基供体, 包括氟代糖、氯代糖、溴代糖和碘代糖. 其中最早得到广泛应用的是溴代糖和氯代糖.

1901 年, Koenigs 和 Knorr ${ }^{[21]}$ 使用全乙酰葡萄糖溴代 物 5 为供体, $\mathrm{Ag}_{2} \mathrm{CO}_{3} / \mathrm{Ag}_{2} \mathrm{O}$ 作为活化剂, 成功实现了与 醇的偶联反应(图 2). 这是糖基化方法发展过程中至关 重要的一步，该方法被命名为 Koenigs-Knorr 反应.
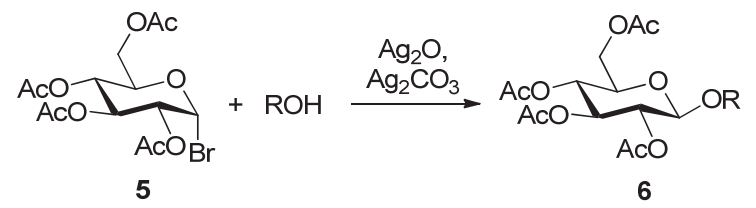

图 2 Koenigs-Knorr 反应

Figure 2 Koenigs-Knorr glycosylation reaction

除了溴代糖, Koenigs-Knorr 反应也可以使用氯代糖 为供体. 早期的时候 Koenigs-Knorr 反应只能使用简单 的醇做受体, 而以亲核性较弱的糖作受体时, 反应效率 不高. 随后人们发现换用活性更高的重金属盐可以提高 氯/溴代糖端基离去基的离去能力, 进而提高反应效率, 比如 Zemplén 和 Helferich 等分别于 1930 年和 1949 年 报道了二价的录盐 $\mathrm{Hg}(\mathrm{OAc})_{2}{ }^{\left[{ }^{[22}\right]}$ 和 $\mathrm{Hg}(\mathrm{CN})_{2} / \mathrm{HgBr}_{2}{ }^{[23]}$ 能 更好地替代 $\mathrm{Ag}_{2} \mathrm{CO}_{3}$. 后续其它重金属盐类活化剂也被 发展起来, 如银盐 $\mathrm{AgClO}_{4}{ }^{[24]} 、 \mathrm{AgOTf}^{[25]} 、 \mathrm{AgNO}_{3}{ }^{[26]}$ 、 铜盐 $\mathrm{Cu}(\mathrm{OTf})_{2}{ }^{[27]}$ 、镉盐 $\mathrm{CdCO}_{3}{ }^{[28]}$ 和有机锌盐 $\mathrm{Zn}\left(p-{ }^{t} \mathrm{BuC}_{6} \mathrm{H}_{4} \mathrm{CO}_{2}\right)_{2}{ }^{[29]}$, 其中 $\mathrm{AgOTf}$ 被证明是最有效的 活化剂之一.

氟代糖是卤代糖中化学性质最稳定的糖基供体, 然
而其早期却很少被应用于糖合成中. 主要原因在于缺少 高效的活化剂，直到 1981 年, Mukaiyama 小组才首次报 道了使用 $\mathrm{SnCl}_{2} / \mathrm{AgClO}_{4}{ }^{[30]}$ 活化氟代糖的例子. 随后其他 一些研究者引入了一系列的活化剂, 使得氟代糖成为受 欢迎的糖基供体之一. 其它一些常用的活化剂包括 $\mathrm{SnCl}_{2} / \mathrm{TrClO}_{4}{ }^{[31]}$ ，二 (环戊二烯基) 金属衍生物 $\mathrm{Cp}_{2} \mathrm{MCl}_{2}{ }^{[32]}, \mathrm{SiF}_{4}{ }^{[33]}, \mathrm{TfOH}^{[34]}$ 等.

相比氟/氯/溴代糖, 碘代糖是一种活性很高的糖基 供体, 不过它也是最不稳定的, 这在很大程度上限制了 碘代糖的应用. 直到 20 世纪末期, 随着制备方式的进 步, 碘代糖作为糖基供体才得到复兴, 有时其高活性会 带来反应时间缩短、效率提高和立体选择性改善等优 点 ${ }^{[35]}$. 通常 $\alpha$-碘代糖在碱性条件下可以高效生成 $\beta$-糖 苷 ${ }^{[36]}$ ，或者使用 “卤代物催化”，在 TBAI (四丁基碘化 铵)和 DIPEA ( $N, N$-二异丙基乙胺)条件下，原位异构为 $\beta$-碘代糖，用于合成 $\alpha$-糖苷 ${ }^{[37]}$.

卤代糖作为一类经典的糖基供体，其使用有时也受 到限制，例如可能会生成原酸酯副产物，高活性卤代糖 不适于长时间存储(如碘代糖一般原位制备), 严重依赖 重金属盐, 等等. 近期我们研究小组及其他研究者使用 有机小分子成功实现了氯/溴代糖活化 ${ }^{[38,39]}$, 克服了其 依赖重金属盐的缺点，这些方法在糖合成中的价值有待 进一步检验. 如何使卤代糖糖基化更加绿色环保仍然值 得继续探索.

\subsection{2 糖基三氯乙酰亚胺酯作为糖基供体}

Schmidt 小组 ${ }^{[15]}$ 于 1980 年首次报道了一种很受欢迎 的糖基供体一一糖基三氯乙酰亚胺酯(Glycosyl trichloroacetimidate, TCAI), 该供体也被称作 Schmidt 供体.

Schmidt 供体可以从半缩醛糖和 $\mathrm{Cl}_{3} \mathrm{CCN}$ 在碱性条 件下制备, 使用 $\mathrm{K}_{2} \mathrm{CO}_{3}$ 有利于生成 $\beta$-型异构体，而使用 $\mathrm{NaH} 、 \mathrm{Cs}_{2} \mathrm{CO}_{3}$ 和 DBU (1,8-二氮杂二环[5.4.0]十一碳-7烯)则倾向于生成 $\alpha$-型异构体(图 3a). 该供体在储藏时有 一定的稳定性, 容易被催化量的路易斯酸(如 TMSOTf 或 $\mathrm{BF}_{3} \cdot \mathrm{Et}_{2} \mathrm{O}$ )所活化, 糖基化反应的效率通常很高(图 $3 b)$. 正是由于这些优点, 糖基三氯乙酰亚胺酯在复杂

a: Preparation of glycosyl trichloroacetimidates (TCAI)

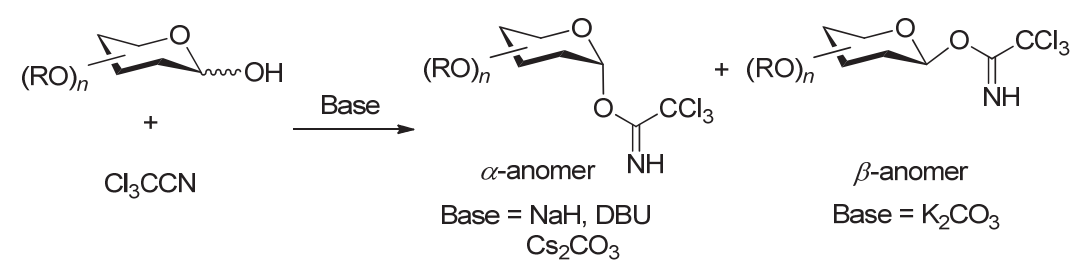

b: Glycosylation reaction with TCAI as donor
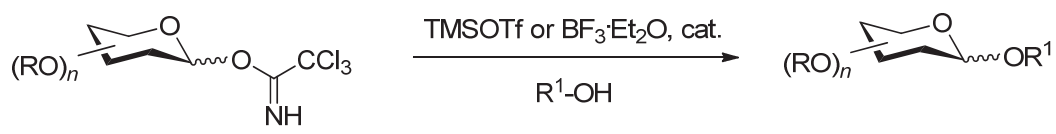

图 3 糖基三氯乙酰亚胺酯的制备及其糖基化反应

Figure 3 Preparation of glycosyl trichloroacetimidates (TCAI) and their glycosylation reactions 
聚糖及糖缀合物的化学合成中应用得十分广泛.

然而, 糖基三氯乙酰亚胺酯也不是完美的糖基供 体，比如当与活性很低的受体反应时，会重排为 $N$-糖基 三氯乙酰胺. 使用反加操作(Inverse procedure) $)^{[40]}$ 一一即 把预先混合的受体与活化剂加至供体中, 有时可以较好 地解决这一问题. 值得注意的是, 某些糖基三氯乙酰亚 胺酯的衍生型供体在某些糖基化反应中表现出更高的 效率, 例如 $\mathrm{Yu}$ 小组 ${ }^{[41]}$ 报道的 $O$-糖基 $N$-苯基三氟乙酰亚 胺酯( $O$-Glycosyl $N$-phenyltrifluoroacetimidate, PTFA)供 体，近期也有比较多的合成应用.

\subsection{3 硫苷作为糖基供体}

硫苷(Thioglycoside)是聚糖合成中另一类明星糖基 供体, 最常用的硫苷为苯硫苷和乙硫苷. 最早使用硫苷 进行糖基化反应可以追溯到 1973 年, Ferrier 小组 ${ }^{[42]}$ 以 $\mathrm{HgSO}_{4}$ 为活化剂, 实现了全茮基葡萄糖苯硫苷与糖受体 之间的偶联反应.

之后, 一系列新的硫苷活化剂被开发出来. 它们主 要可以被分为四类, 如图 4 所示.

第一类是金属盐类活化剂. 早期的例子主要是录 盐、铜盐和银盐, 这类活化剂使用时通常需要比较苛刻 的条件, 现已很少使用.

第二类是类卤鎓离子亲电试剂. 其中最常用的是 van Boom 和 Fraser-Reid 分别报道的 NIS/TfOH ${ }^{[43]}$ 和 $\mathrm{NIS} / \mathrm{AgOTf}{ }^{[44]}$, 这两种活化剂都可以直接买到, 活化能 力很强并且副反应较少, 因而在硫苷活化中用得很广 泛.

第三类是有机硫试剂, 这类活化剂有 Garegg 首先 使用的二甲基甲硫基硫鎓三氟甲磺酸盐(DMTST) ${ }^{[45]}$, Whitesides 发展的 $\mathrm{PhSCl} / \mathrm{AgOTf}{ }^{[46]}$, Crich 发展的苯基哌

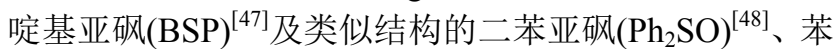

基吗啉基亚砜 (BSM) ${ }^{[49]}$ 与三氟甲磺酸酐的组合 $\left(\mathrm{BSP} / \mathrm{Tf}_{2} \mathrm{O}, \mathrm{Ph}_{2} \mathrm{SO} / \mathrm{Tf}_{2} \mathrm{O}, \mathrm{BSM} / \mathrm{Tf}_{2} \mathrm{O}\right)$ 等.

第四类是单电子转移试剂及光、电活化方法, 如

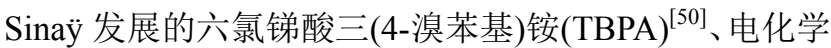
法 $^{[51]}$ 及近年来不断兴起的光化学法 ${ }^{[52]}$ 等.

硫苷的活化一般需要当量的活化剂, 不过近期也有 研究者报道亚当量的活化剂也能完全活化硫苷. 2013 年, Pohl 小组 ${ }^{[53]}$ 发现使用 $\mathrm{Ph}_{3} \mathrm{Bi}(\mathrm{OTf})_{2}$ 可以在室温下活化 硫苷，该活化剂具有溶解性好、对空气/光稳定并且半衰 期长等优点, 并且有些例子只需要 0.5 equiv.活化剂即可 完全活化硫苷供体.

总之，由于硫苷制备非常方便，在许多功能基转化 条件下也足够稳定, 而且可以很容易被软亲电试剂活 化，因此使用硫苷进行糖基化反应一直受到重视，不仅 新的活化剂或活化方式不断地被报道，而且有关硫苷用 于复杂糖类化合物合成的工作也大量发表. 更重要的 是, 硫苷既可以作为端基保护基、又可以作为优良离去 基的性质，赋予了它在聚糖类化合物合成中独特的价 值，使它尤其适用于聚糖的液相一釜多步合成.

\section{3 聚糖的组装策略}

使用现有的糖基化方法，探索如何高效地将糖砌块 组装在一起, 进而形成复杂的聚糖结构, 这是糖化学中 另一个重要的问题，即聚糖的组装策略问题.

传统的聚糖组装策略主要有两种类型. 第一种是线 性合成，按照糖链合成的延伸方向，又可以分为从非还 原端到还原端(图 5a)和从还原端到非还原端(图 5b)两种 类型; 第二种是汇聚式合成，一般是指先通过线性合成 得到一些短的糖片段，最后再进行糖片段之间的偶联. 汇聚式合成可以实现分子尺寸的快速增加.

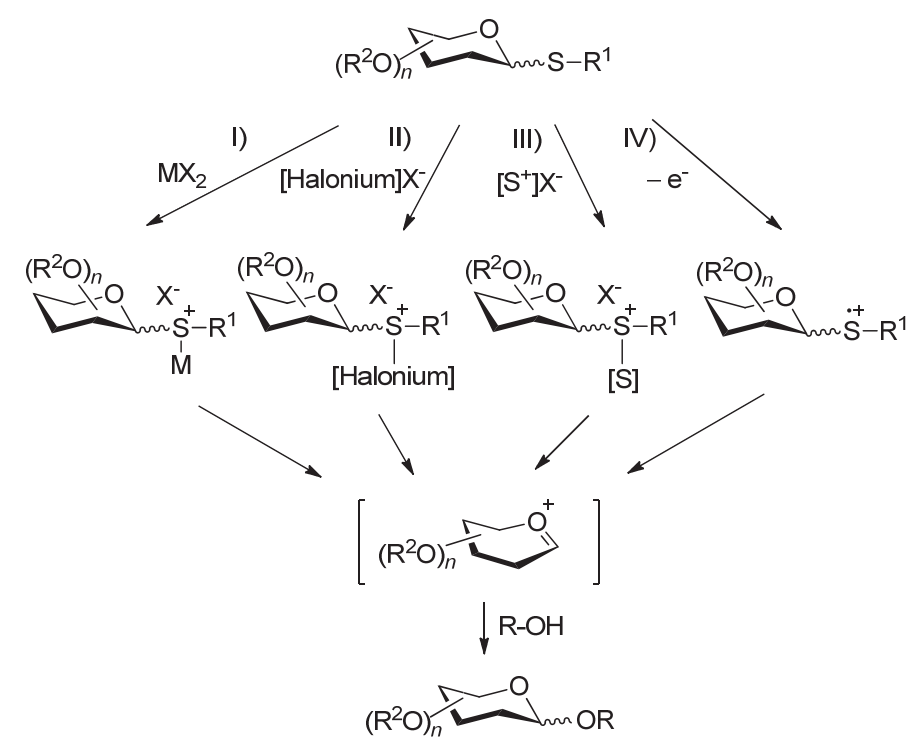

图 4 常见的硫苷活化剂分类

Figure 4 Different types of promoters for thioglycosides 


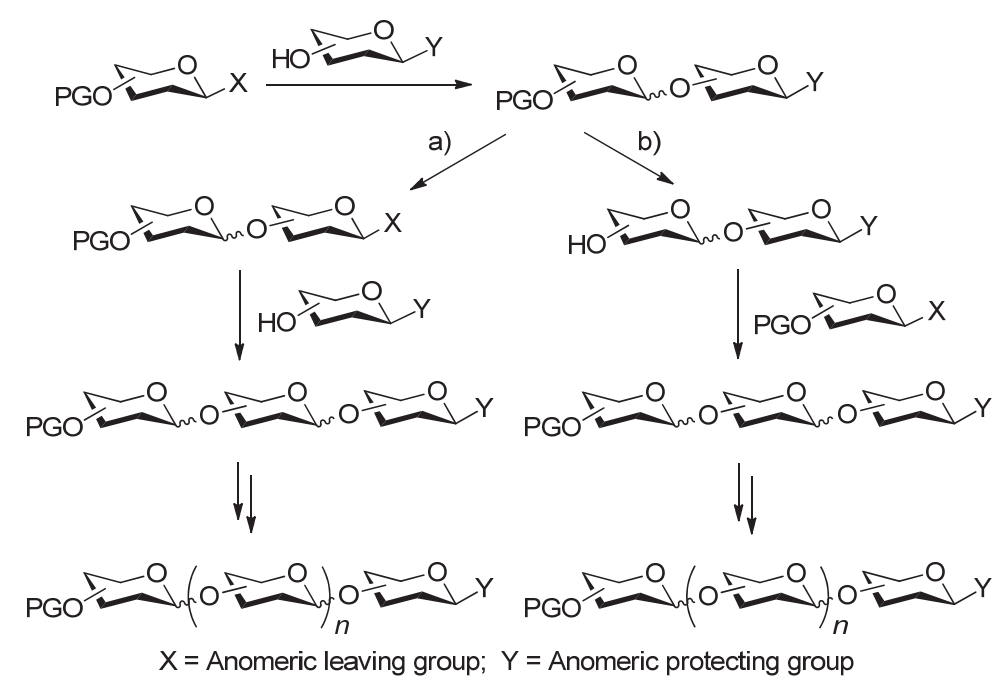

图 5 糖链的线性合成策略

Figure 5 Linear synthesis strategy for glycan assembly

无论是线性合成还是汇聚式合成，通常都会涉及到 端基取代基的不断调整或糖上临时保护基的反复脱除, 这就使得整体合成步骤一般较长, 需要对中间体分离纯 化, 最终降低了聚糖合成的效率.

为了提高聚糖组装的效率, 近三十年来研究者们发 展了不少新策略. 其中最具代表性的工作包括正交选择 性的液相一釜合成策略 ${ }^{[54]}$ 、基于糖基供体活性的程序化 液相一釜合成策略 ${ }^{[55]}$ 、预活化液相一釜合成策略 ${ }^{[56]}$ 和自 动化固相合成策略 ${ }^{[57]}$.

\section{1 正交选择性的液相一釜合成策略}

液相一釜合成策略一般是在同一个反应容器中, 实 现多个糖砌块从非还原端向还原端的有序偶联, 最终通 过一次纯化步骤即可快速实现目标聚糖链的组装. 这类 策略避免了传统聚糖链合成中反复的保护/去保护操作, 也不需要对中间体进行分离, 从而使聚糖合成步骤大大 简化.

正交选择性的液相一釜合成策略是通过选用不同 的活化剂, 实现多个带有不同离去基的糖砌块的正交选 择性活化, 从而在同一个反应容器中完成多个糖苷键的 构建(图 6).
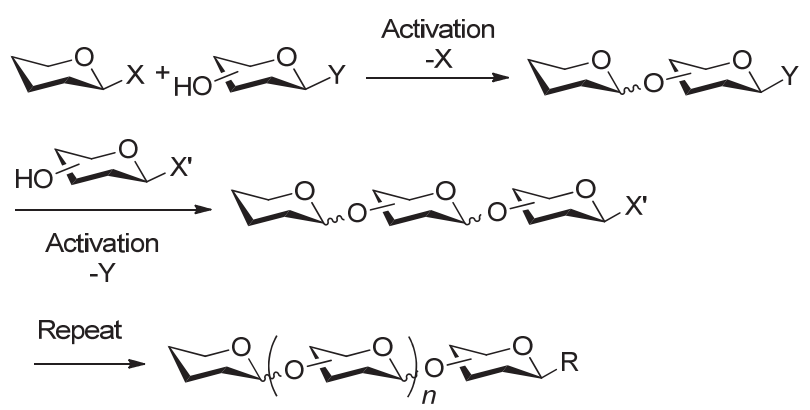

图 6 正交选择性的液相一釜合成策略

Figure 6 Orthogonal selective one-pot glycosylation strategy in solution phase
该策略最早由 Takahashi 小组 ${ }^{[54]}$ 于 1994 年提出, 随 后被成功应用于多种聚糖如植物抗毒素诱导剂七糖 ${ }^{[58]}$ 、 薯蓣㿝苷类化合物 ${ }^{[99]}$ 、黏蛋白核心分支七糖 ${ }^{[60]}$ 、糖氨基

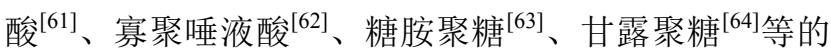
化学合成中. 然而该策略也存在一定缺陷, 即为了实现 正交活化，至少需要制备两套带不同离去基的糖砌块， 这给原料的制备增加了困难，而且目前能够实现正交活 化的离去基组合也还比较有限.

\section{2 基于糖基供体活性的程序化液相一釜合成策略}

基于糖基供体活性的程序化液相一釜合成策略是 使用一套带相同离去基的糖基模块, 按照其反应活性的 高低依次加入到反应瓶中，使糖基化反应按照设计好的 方向有序进行(图 7). 其基本原理是只要相邻两个模块 活性差别足够大，活性较高的糖基供体就会与活性较低 的糖模块发生糖基化反应，而不会发生活性较低的糖模 块间的自身偶联.

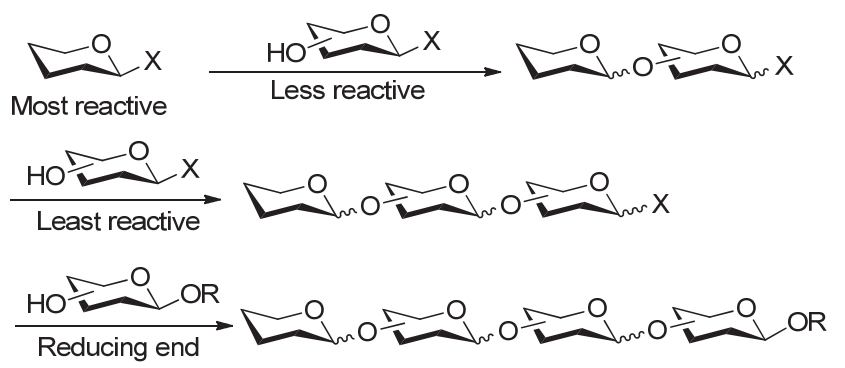

图 7 基于糖基供体活性的程序化液相一釜合成策略

Figure 7 Reactivity-based one-pot glycosylation strategy in solution phase

该策略由 Wong 小组 ${ }^{[55]}$ 在 1999 年提出, 目前已经被 成功应用于一些肿瘤相关糖抗原 Globo- $\mathrm{H}^{[65]}$ 、 Lewis $\mathrm{X}$ 二聚体 ${ }^{[66]} 、$ Lewis $Y^{[67]} 、 N 3$ minor 八糖 ${ }^{[68]}$ 、岩藻糖化的

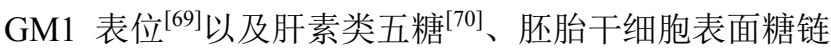
Lc4 和 IV2Fuc-Lc4 $4^{[71]}$ 等重要寡糖分子的合成. 不过, 由 
于该策略对糖基模块活性差别的依赖很大，目前最多实 现四个组分的一釜合成; 并且由于糖模块活性需要通过 精细的保护基操作来调节, 使得糖模块制备比较繁琐, 这在很大程度上限制了该策略的进一步应用.

\section{3 预活化液相一釜合成策略}

预活化液相一釜合成策略由我们小组和 Huang 小 组于 2004 年共同提出 ${ }^{[56]}$, 其基本原理如图 8 所示: 单糖 供体(一般为对甲基苯硫苷)在低温下用促进剂预先活 化, 生成活性中间体(Active intermediate), 随后加入带 相同离去基的单糖受体进行反应, 即可完成二糖的偶 联; 二糖又可以作为供体与下一个带相同离去基的受体 偶联, 这样多次重复供体预活化和加入受体进行糖基化 的操作, 即可实现目标聚糖链的快速合成.

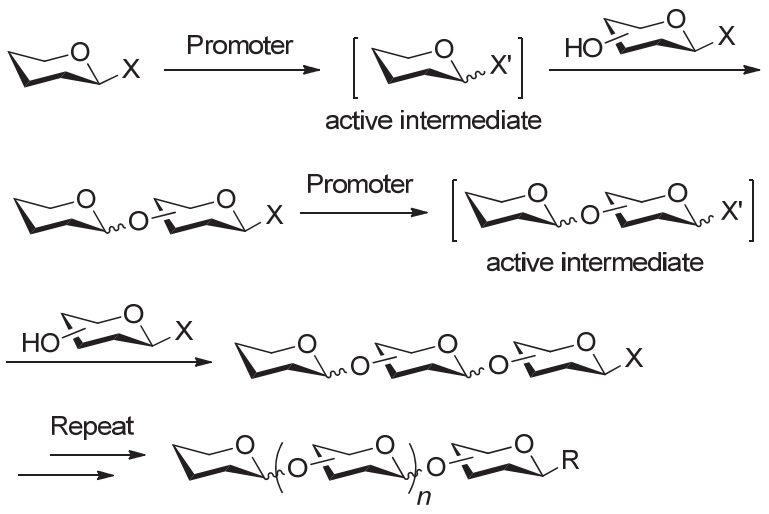

图 8 预活化液相一釜合成策略

Figure 8 Preactivation-based one-pot glycosylation strategy in solution phase

在预活化一釜合成策略中, 所有糖基供体和受体 都使用相同的离去基和活化条件, 只通过加料顺序的改 变就能实现聚糖链的有序延长, 而且能够实现低活性供 体与高活性受体的偶联, 克服了程序化一釜合成策略对 糖模块反应活性的依赖. 该策略的成功应用包括肿瘤抗 原 Globo- $\mathrm{H}^{[72]}$ 和 $\mathrm{KH}-\mathrm{1}^{[73]}$ 、透明质酸寡糖 ${ }^{[74]}$ 、Lewis $\mathrm{X}^{[75]}$ 、

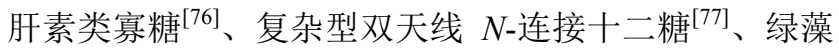
衣壳蛋白新型 $N$-glycan ${ }^{[78]}$ 以及多种细菌表面糖链 ${ }^{[79]}$ 的 合成等.

不过该策略目前也还存在一些不足, 比如有时候会 出现苷元转移, 因此仍然需要发展新的偶联方法来提高 单步糖基化反应的偶联效率.

\section{4 自动化固相合成策略}

液相一釜合成策略通过在同一个反应瓶中快速完 成多步糖基化反应，最后只进行一次分离纯化，从而大 大提高糖合成效率; 而自动化固相合成策略与之不同, 其反应在固相载体上进行, 通过载体的辅助完成中间体 的快速纯化并且使用机器来提高聚糖合成效率.

Seeberger 小组 ${ }^{[57]}$ 于 2001 年首先报道了聚糖的自动 化固相合成技术. 与多肽或核酸的自动化合成方法类似，
他们通过一个连接臂(Linker)将糖受体连接在树脂上, 每步糖基化反应完成后，脱除临时保护基再进行下一步 反应，中间体的纯化通过简单的过滤和洗涤即可快速完 成, 最后再将目标糖链从树脂上切下来(图 9).

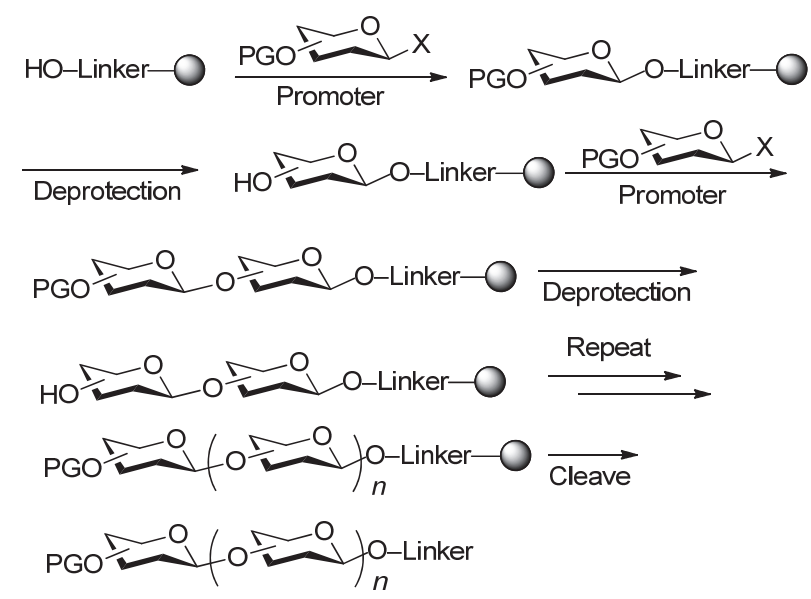

图 9 自动化固相合成策略

Figure 9 Automated solid phase glycosylation strategy

使用自动固相合成组装聚糖链，一般只需要在最后 一步借助柱层析分离，而且由于每次糖基化可以使用大 大过量的糖基供体以确保负载在树脂上的糖基受体尽 量反应完全，因而整体效率通常都比较高.

Seeberger 及其他小组将自动化固相合成策略用于 多种聚糖结构的合成, 包括植物抗毒素诱导子十二 糖 ${ }^{[57]}$ 、肿瘤相关糖抗原 Globo- $\mathrm{H}^{[80]}$ 和 $\mathrm{Le}^{\mathrm{y}}-\mathrm{Le}^{\mathrm{x}}(\mathrm{KH}-1)^{[81]}$ 、 $N$-glycan 的核心五糖 ${ }^{[82]} 、 \beta$-甘露聚糖 ${ }^{[83]}$ 、糖胺聚糖 ${ }^{[84]}$ 以及糖基磷脂酰肌醇(Glycosylphosphatidylinositol, GPI) 六糖等 ${ }^{[85]}$.

不过，该策略也存在一些不足之处，例如: (1) 由于 糖基受体与树脂相连导致反应活性降低，因此每步糖基 化反应需要加入多达 10 equiv.的供体以确保受体尽量完 全消耗，这对于本身就需要多步反应才能制备的糖基模 块是极大的消耗，因而花费较大; (2) 对于比较难构建 的糖苷键如 1,2-顺式糖苷键，由于缺乏可靠的制备方法， 因此往往需要使用提前制备好的二糖模块来替代; (3) 随着聚糖链的不断延长, 产物与片段缺失的序列很难分 离，因而通常还需要引入额外的分离纯化策略.

\section{4 多糖的化学合成}

在过去几十年里, 糖化学家创造性地发展了一系列 新颖的糖基供体和相应的活化方式, 极大地丰富了聚糖 化学合成的工具箱; 另一方面，基于这些糖基化方法以 及对糖基化反应理解的不断深入，发展了一些高效的聚 糖组装策略，这使得聚糖合成效率得到了极大的提高. 可以说，就像小分子天然产物全合成 “没有合成不出来 的分子”一样，对于大多数长度适当的寡糖结构，人们 都有办法完成其化学合成. 
然而, 结构复杂的长链多糖的化学合成对糖化学家 而言仍然是一项富有挑战的任务. 事实上, 迄今为止, 人们完成的复杂长链多糖的化学合成工作也为数不多, 接下来我们将结合多糖合成代表性的例子进行介绍.

\section{1 动物来源多糖的化学合成}

早在 1993 年, Ogawa 小组 ${ }^{[86]}$ 报道了一例来源于兔红 细胞膜的复杂多糖 7 的化学合成, 该分子由 25 个单糖单 元组成, 是一种含有多个 $N$-乙酰乳糖胺的五天线型多 糖(图 10). 作者设计了高度汇聚式的合成策略, 希望最 后通过一步糖基化反应将 5 个三糖供体 8 和一个含 5 个 裸露差基的十糖受体 $\mathbf{9}$ 偶联在一起. 氟代糖供体 $\mathbf{8}$ 可以 拆分为硫苷供体 10 和还原端 PMP (4-甲氧基苯基)保护 的二糖受体 11. 十糖受体 9 可以由氟代糖 12 与已知寡 糖化合物 13 反应再脱除临时保护基 Lev 得到; 而化合 物 12 则可以继续拆分为三个二糖模块 14, 15 和 16. 在 这个工作中, 除了供体 $\mathbf{1 0}$ 外, 所有的糖偶联反应都以氟 代糖为供体. 汇聚式合成策略的使用, 使得线性步骤大 为缩短. 但在糖模块的线性合成过程中, 多次涉及到临 时保护基的脱除以及将端基 PMP 保护基调整为离去基 $\mathrm{F}$, 因而总的步骤仍然较长, 这在一定程度上降低了整 体合成效率.

近期, Gardiner 小组 ${ }^{[87]}$ 报道了他们在多糖化学合成 中的最新进展. 他们以四糖为基本模块, 使用从还原端 到非还原端的线性合成策略, 合成了一系列长度从 16-mer 到 40-mer 的肝素类多糖. 如图 11 所示, 他们以 带一个临时保护基 $\mathrm{ClAc}$ 的十二糖 17 为起始原料, 脱除 ClAc 后得受体 18 , 再与四糖硫苷供体 19 在 NIS/AgOTf

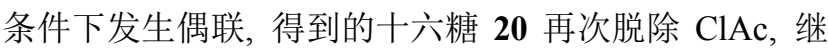
续与四糖硫苷 19 偶联, 之后不断重复脱除临时保护基/ 偶联过程，最终可以得到最长为四十糖的全保护肝素类 多糖.

需要说明的是, 在该工作中, 作者只对最长为二十 糖的聚糖链 21 进行了脱保护和官能团化, 得到 $N$-硫酸 化和部分 $O$-硫酸化的二十糖 22. 即便如此, 聚糖 22 也 已经是人工合成的结构均一的最长肝素类多糖(图 12).

\section{2 植物来源多糖的化学合成}

2005 年, Kong 小组 ${ }^{[88]}$ 报道了一例植物来源多糖 $\mathbf{2 3}$ 的化学合成工作(图 13). 该多糖属于阿拉伯半乳聚糖, 存在于具有免疫调节活性的紫花紫雉菊(Echinacea purpurea)中, 由一条 $\beta-1,6$ 连接的半乳糖主链和若干 $\alpha-1,2$ 、 $\alpha-1,3$ 连接的 $L$-呋喃阿拉伯糖分支组成.

作者设计合成了多个单糖砌块 27, 28, 29, 30 和 31, 这些砌块使用乙酰基(Ac)或者烯丙基(All)作为临时保护 基，其余位置均用 $\mathrm{Bz}$ 保护，糖苷键的构建主要使用糖 基三氯乙酰亚胺酯和硫苷作为供体. 首先从单糖砌块出 发，经多步反应合成了三个四糖片段 $24 、 25$ 和 26; 再以 这些四糖为模块，从还原端向非还原端延长糖链；最后 再进行整体脱保护操作即得目标二十糖 23.
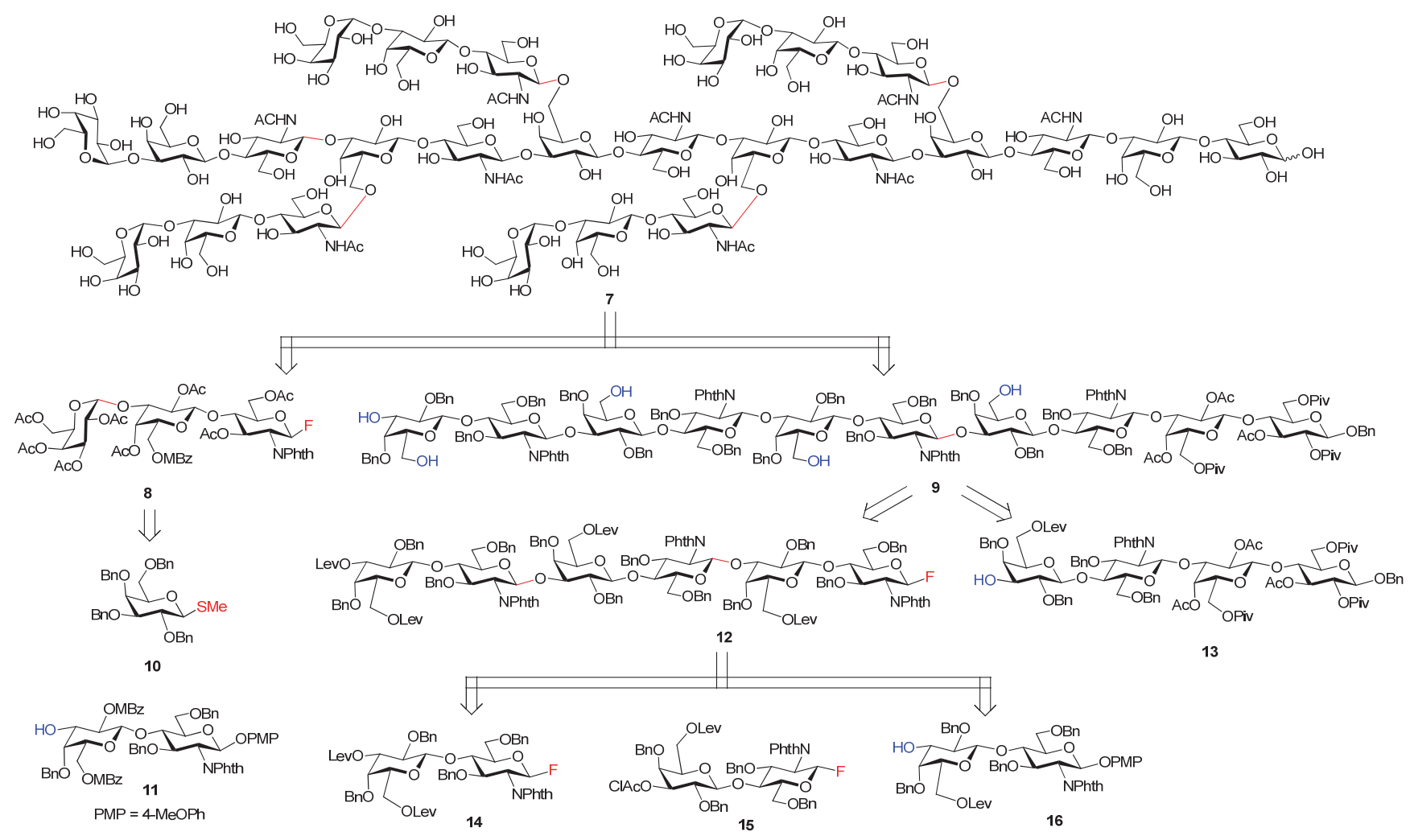

图 10 Ogawa 小组化学合成兔红细胞膜多糖 7

Figure 10 Ogawa's chemical synthesis of polysaccharide 7 from rabbit erythrocyte membrane 

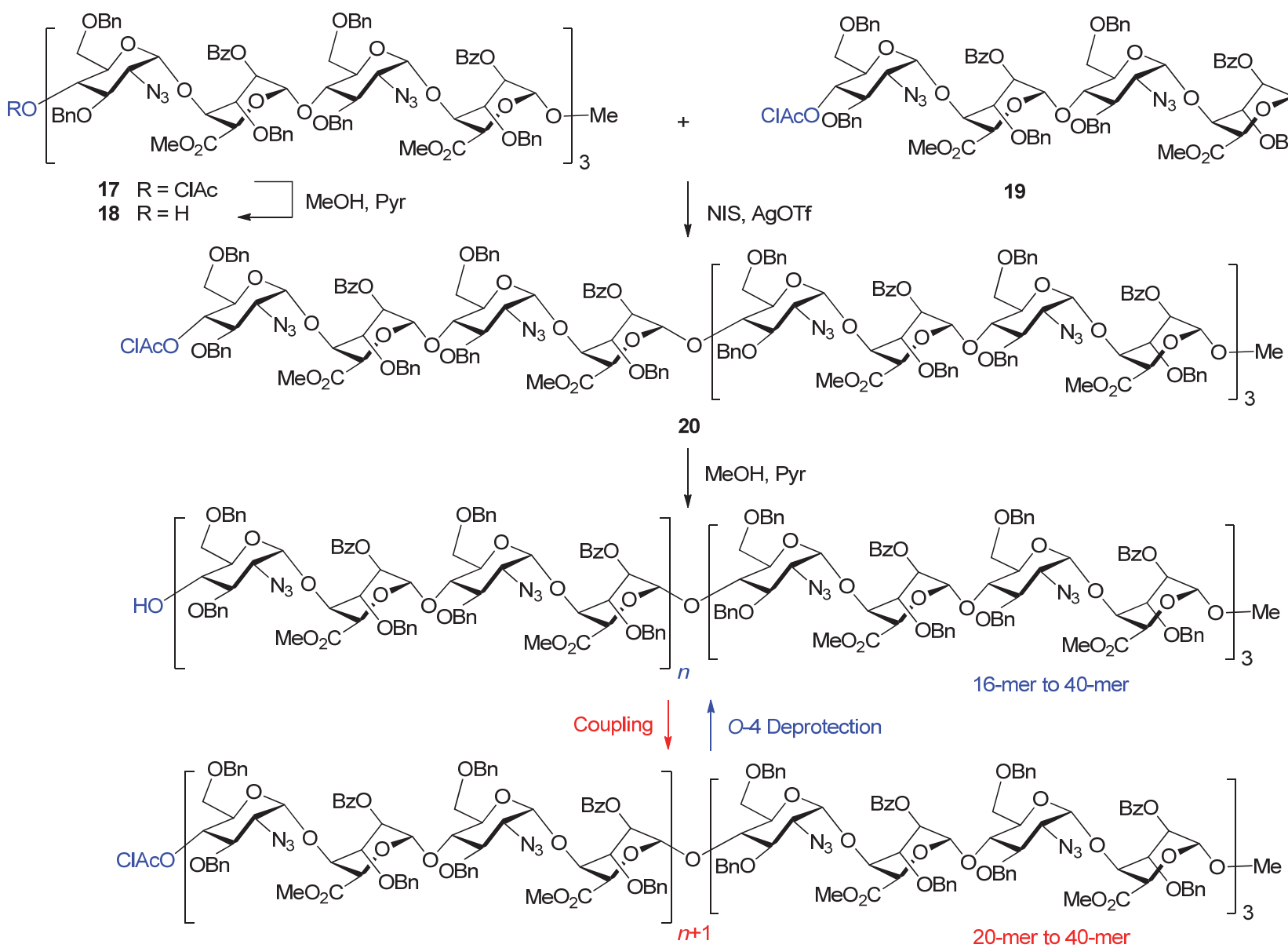

图 11 Gardiner 小组化学合成肝素类多糖工作

Figure 11 Gardiner's work on chemical syntheses of heparin-related polysaccharides

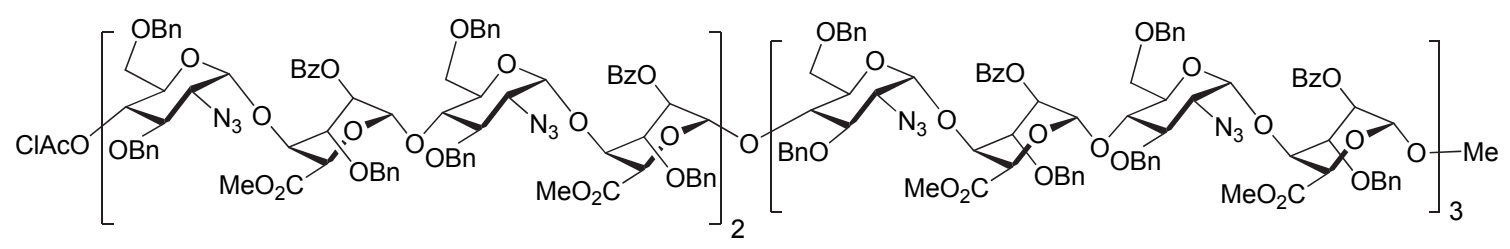

21

a) $\mathrm{LiOH}, \mathrm{THF} / \mathrm{MeOH} / \mathrm{H}_{2} \mathrm{O}, 48 \%$ c) $\mathrm{Pd}(\mathrm{OH})_{2} / \mathrm{C}, \mathrm{EtOH} / \mathrm{H}_{2} \mathrm{O}, \mathrm{H}_{2}, 89 \%$

b) $\mathrm{NMe}_{3} \cdot \mathrm{SO}_{3}, \mathrm{DMF}, 90 \%$ d) Pyridine $\mathrm{SO}_{3}$ complex, $\mathrm{H}_{2} \mathrm{O}$

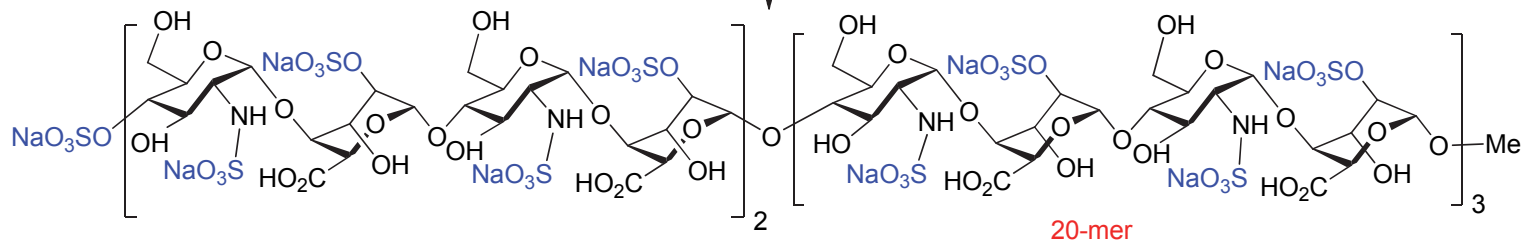

22

图 12 对全保护多糖 21 进行脱保护和硫酸化制备肝素类多糖 22

Figure 12 Deprotection and sulfation of fully protected polysaccharide 21 to afford heparin-like polysaccharide 22

\section{3 细菌来源多糖的化学合成}

细菌细胞壁往往由丰富的多糖结构组成, 这些多糖 往往与细菌的存活力和致病力有关, 其生物合成酶往往
是一些抗生素的作用靶标. 另外，一些抗原性多糖(或 其蛋白缀合物)能刺激机体产生保护性抗体，常常被开 发成预防性疫苗. 因此, 细菌来源的多糖吸引了很多糖 化学家的合成兴趣. 


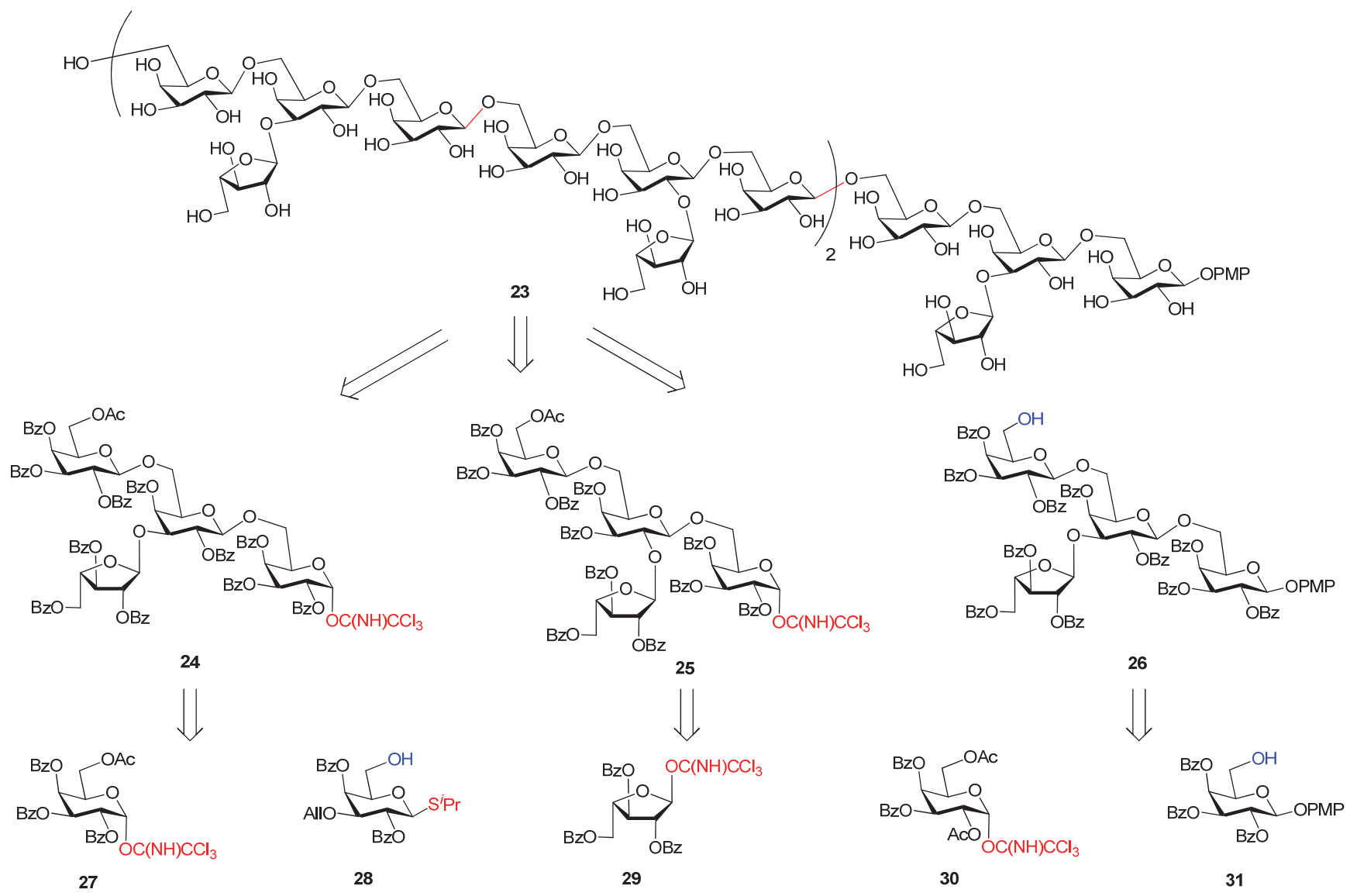

图 13 Kong 小组化学合成植物来源多糖 $\mathbf{2 3}$ 的工作

Figure 13 Kong's work on the chemical synthesis of plant polysaccharide 23

Pozsgay 小组 ${ }^{[89]}$ 早在 20 世纪末就致力于痢疾志贺氏 菌糖疫苗的研究, 他们于 1998 年报道了痢疾志贺氏菌 内毒素 $O$-抗原四糖重复序列的一个四聚体 32 的合成. 如图 14 所示, 作者从四糖受体 $\mathbf{3 3}$ 出发, 以相同骨架的 四糖三氯乙酰亚胺酯 34 为模块, 通过模块之间的糖基 化偶联反应快速延长糖链, 接着脱除临时保护基用于下 一步糖基化, 最终高效地合成了十六糖 32. 值得一提的 是, 作者在后续的活性评价中发现, 合成得到的多糖与 载体蛋白缀合后, 所引起的血清抗体滴度比天然来源的 多糖-蛋白缀合物更高 ${ }^{[00]}$.

在另一个工作中, Pozsgay 等 ${ }^{[91]}$ 采用类似的 “模块 化” 策略继续延长糖链, 最终完成了一个二十四糖 35 的合成(图 15). 为了方便在糖基化反应完成后快速分离 目标产物, 作者在还原端的四糖模块 36 上引入了多个 疏水性长链烷烃, 这样在合成过程中, 只需将产物吸附 在反相硅胶上, 之后用廉价低毒的醇类溶剂洗脱就可以 完成目标产物的纯化.

结核分枝杆菌细胞壁中富含结构独特的多糖结构, 其中阿拉伯半乳聚糖(Arabinogalactan, $\mathrm{AG}$ )得到了较多 关注, 该多糖全部由呋喃型糖组成, 这类单糖不存在于
任何哺乳动物体内, 而且含有多个合成难度较大的 1,2顺式呋喃阿拉伯糖苷键. 化学合成得到的阿拉伯半乳聚 糖(或其片段)可用于阐明结核分枝杆菌细胞壁生物合成 途径，进而有助于研制新型抗结核药物; 同时也有开发 成预防结核病的新型糖疫苗的潜力.

2007 年和 2011 年, Lowary ${ }^{[92]}$ 和 Ito $^{[93]}$ 小组分别完成 了两条几乎相同的阿拉伯聚糖 37 和 $\mathbf{3 8}$ 的化学合成(图 16 和图 17). 这两个多糖都由 22 个呋喃阿拉伯糖组成 $\left(\operatorname{Ara} f_{22}\right)$, 有 3 处分支位点, 且非还原端含 4 个 $\beta-1,2$ 糖苷 键.

在 Lowary 小组的全合成工作中, 合成短的糖模块 时使用对甲苯硫苷为供体，而在片段偶联阶段选用糖基 三氯乙酰亚胺酯作为供体, 另外, 除临时保护基选用 TBDPS 或者 Lev 外, 其余位点都用 Bz 保护以方便最后 一步整体去保护.

作者首先合成了三种主要的糖片段 $41 、 45$ 和 48 . 分 支五糖 48 的两个 $\beta-1,2$ 呋喃阿拉伯糖苷键是使用 3,5-O-di-tert-butylsilylidene (DTBS) 保护的单糖供体构 建的. 将这些片段依次拼接在一起, 最后脱除 $\mathrm{Bz}$ 和还 原 $\mathrm{N}_{3}$, 即得到目标多糖 37(图 16). 

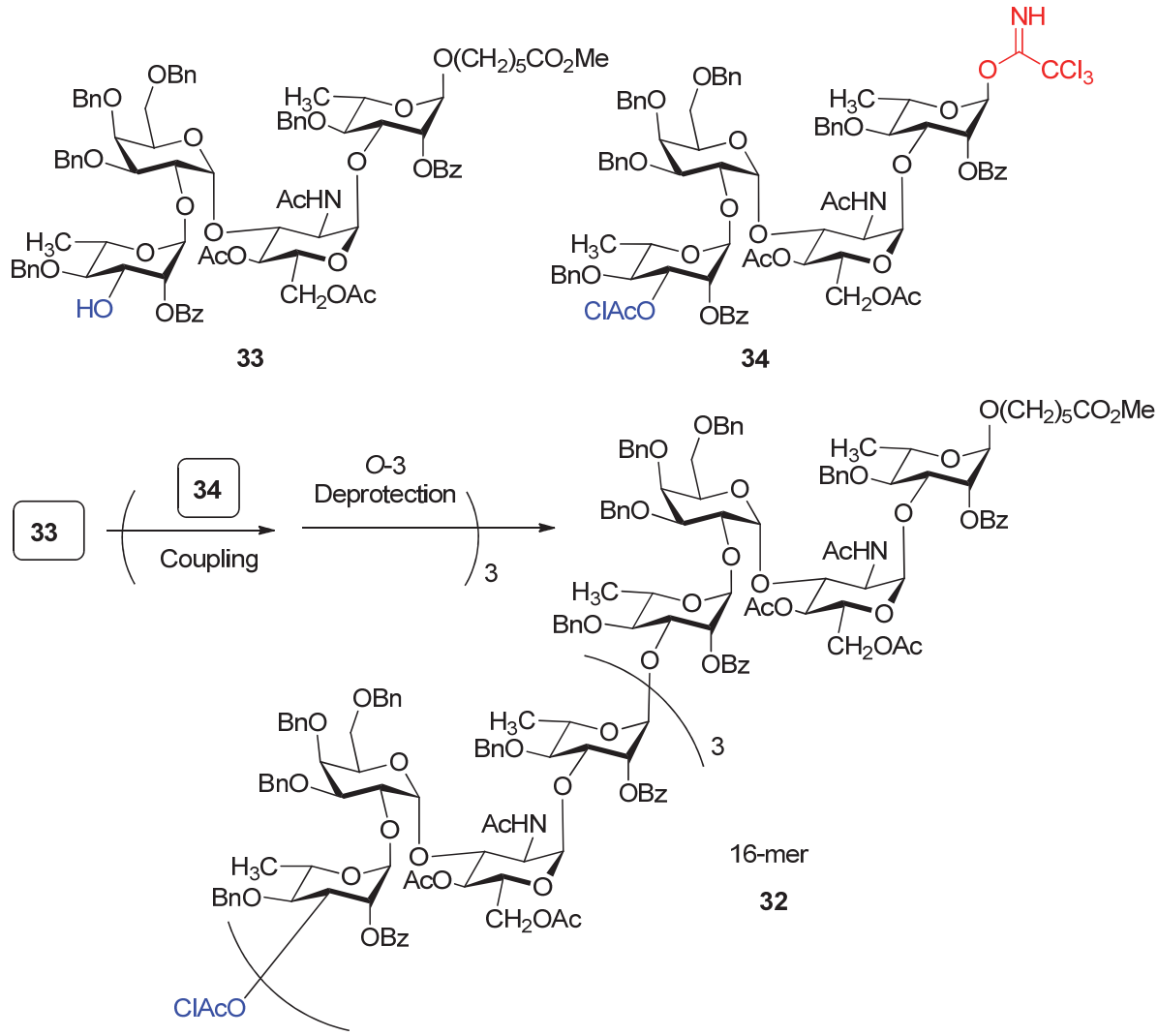

图 14 Pozsgay 小组化学合成痢疾志贺氏菌 $O$-抗原多糖 $\mathbf{3 2}$

Figure 14 Pozsgay's chemical synthesis of the $O$-specific polysaccharide 32 of Shigella dysenteriae
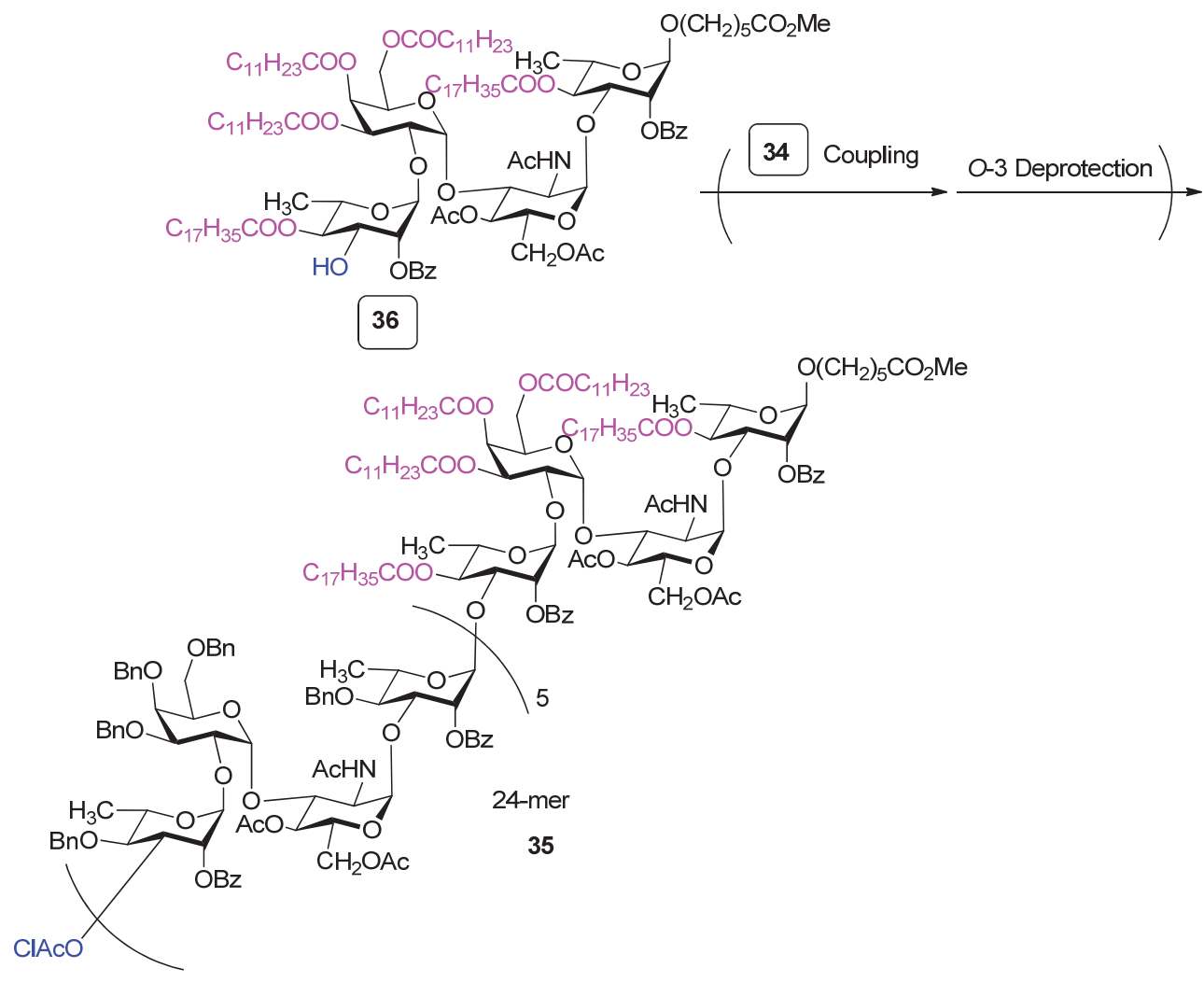

图 15 Pozsgay 小组化学合成痢疾志贺氏菌 $O$-抗原多糖 $\mathbf{3 5}$

Figure 15 Pozsgay's chemical synthesis of the $O$-specific polysaccharide 35 of Shigella dysenteriae 


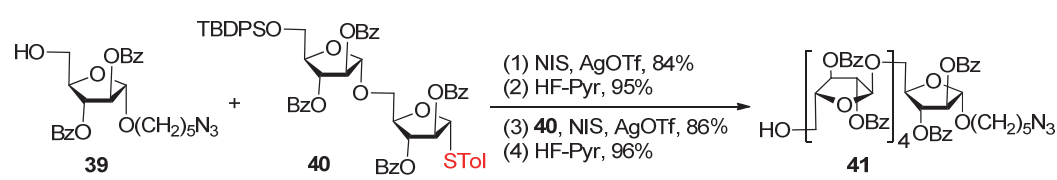

39

41
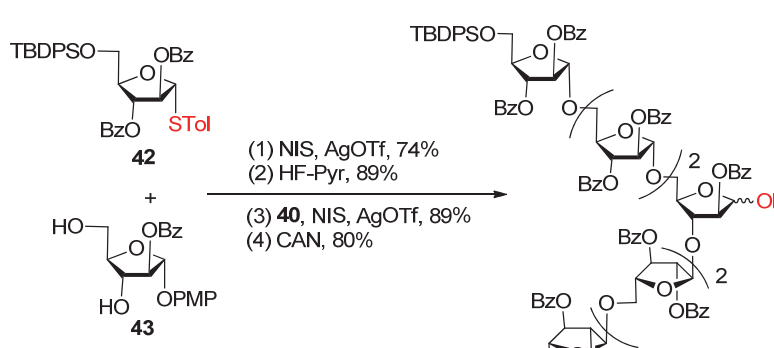

(2) 41, TMSOTf,

$77 \%$ (2 steps)
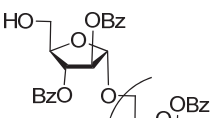

$20 \mathrm{~B}$

2 OBz [ OBzO

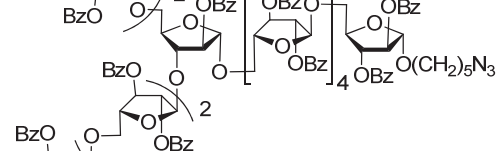
<smiles>CCCOC1COC2CCOC1CO2</smiles>

45

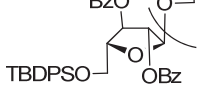

44

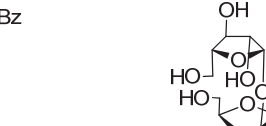

tor

45
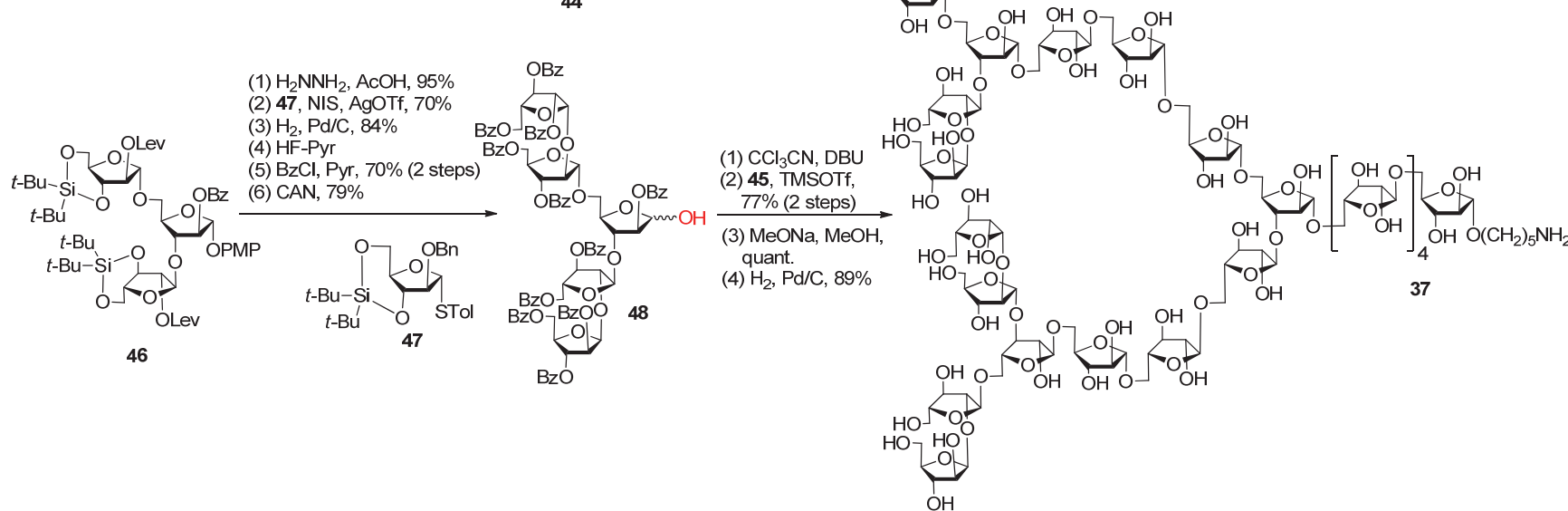

图 16 Lowary 小组化学合成结核分枝杆菌细胞壁多糖 $\mathbf{3 7}$

Figure 16 Lowary's chemical synthesis of polysaccharide $\mathbf{3 7}$ of Mycobacterium tuberculosis cell wall

Ito 在合成二十二糖 $\mathbf{3 8}$ 时, $\mathrm{Bn}$ 是主要的保护基类型, 而且所有糖苷键的生成都是使用对甲苯硫苷为糖基供 体(图 17). 作者首先将整个分子拆分为三个糖片段 49、

50 和 51, 而单糖供体 52 和 53 则用于立体选择性构建 $\beta-1,2$ 呋喃阿拉伯糖苷键. 使用环状硅保护基 TIPDS 保 护的硫苷 52 可以与受体 49 直接糖基化得到 $\beta$-立体构型 为主的产物, 而使用 Nap 介导的分子内苷元转移(以硫 苷 53 为糖基供体), 则可以得到 $\beta$-立体构型唯一的产物. 与 Lowary 小组合成多糖 $\mathbf{3 7}$ 时需多次将端基 PMP 转化 为三氯乙酰亚胺酯不同的是, Ito 等的合成是将还原端 1,2-异丙叉基通过 “三步法” 转化为对甲苯硫苷.

近期, Hotha 小组 ${ }^{[94]}$ 报道了他们在结核分枝杆菌细 胞壁阿拉伯半乳聚糖化学合成中的进展. 如图 18 所示, 目标分子含有两个呋喃半乳糖, 而不含非还原端的 $\beta-1,2$ 糖苷键. 同以上几个多糖合成类似，作者也采用了 模块化的汇聚式合成策略. 而与 Lowary 或 Ito 使用糖基 三氯乙酰亚胺酯或者硫苷为供体不同的是, 该工作中所 有糖苷键的构建均采用 Hotha 小组自己发展的 $\mathrm{AuCl}_{3} / \mathrm{AgOTf}$ 共催化的以炔丙基原酸酯为供体的糖基化
方法. 最终以 47 步反应，0.09\%的总收率完成了二十一 糖 52 的全合成.

另外, Hotha 小组 ${ }^{[5]}$ 最近还报道了一例阿拉伯半乳 聚糖中二十五糖片段的合成, 其结构与 $\mathbf{5 2}$ 比较类似, 唯 一的差别在于非还原端含有 4 个 $\beta-1,2$ 连接的单糖残基, 并且使用的是该组近期发展的糖基碳酸酯 ${ }^{[96]}$ 为供体.

结核分枝杆菌细胞壁多糖 Arabinogalactan (57, 图 19)的首次全合成是由我们小组 ${ }^{[79 e]}$ 于 2017 年完成的. 完 整的 Arabinogalactan 由一条含 30 个呋喃半乳糖的线性 主链和两条相邻的含 31 个呋喃阿拉伯糖的分支侧链组 成. 该分子共有 8 处分支位点, 且非还原端含有 8 个化 学上难以构建的 $\beta-1,2$ 呋喃阿拉伯糖苷键.

如图 20 所示, Arabinogalactan (57)首先被拆分为两 条多糖链, 即 $\mathrm{Ara}_{31}$ 供体 $\mathbf{5 8}$ 和 $\mathrm{Gal} f_{30}$ 受体 59. $\mathrm{Araf}_{31}$ 供体 58 可以采用预活化液相一釜合成策略，由寡糖链 60,61 和 63 合成得到. 类似地, $\mathrm{Gal}_{30}$ 受体 59 可以由寡糖链 65 , 66,69 和 67 经过一釜五组分糖基化反应合成. 目标分子 非还原端的 $\beta-1,2$ 呋喃阿拉伯糖苷键由环状硅保护的硫 苷供体 71 和二醇硫苷受体 73 在预活化操作下高效构建. 

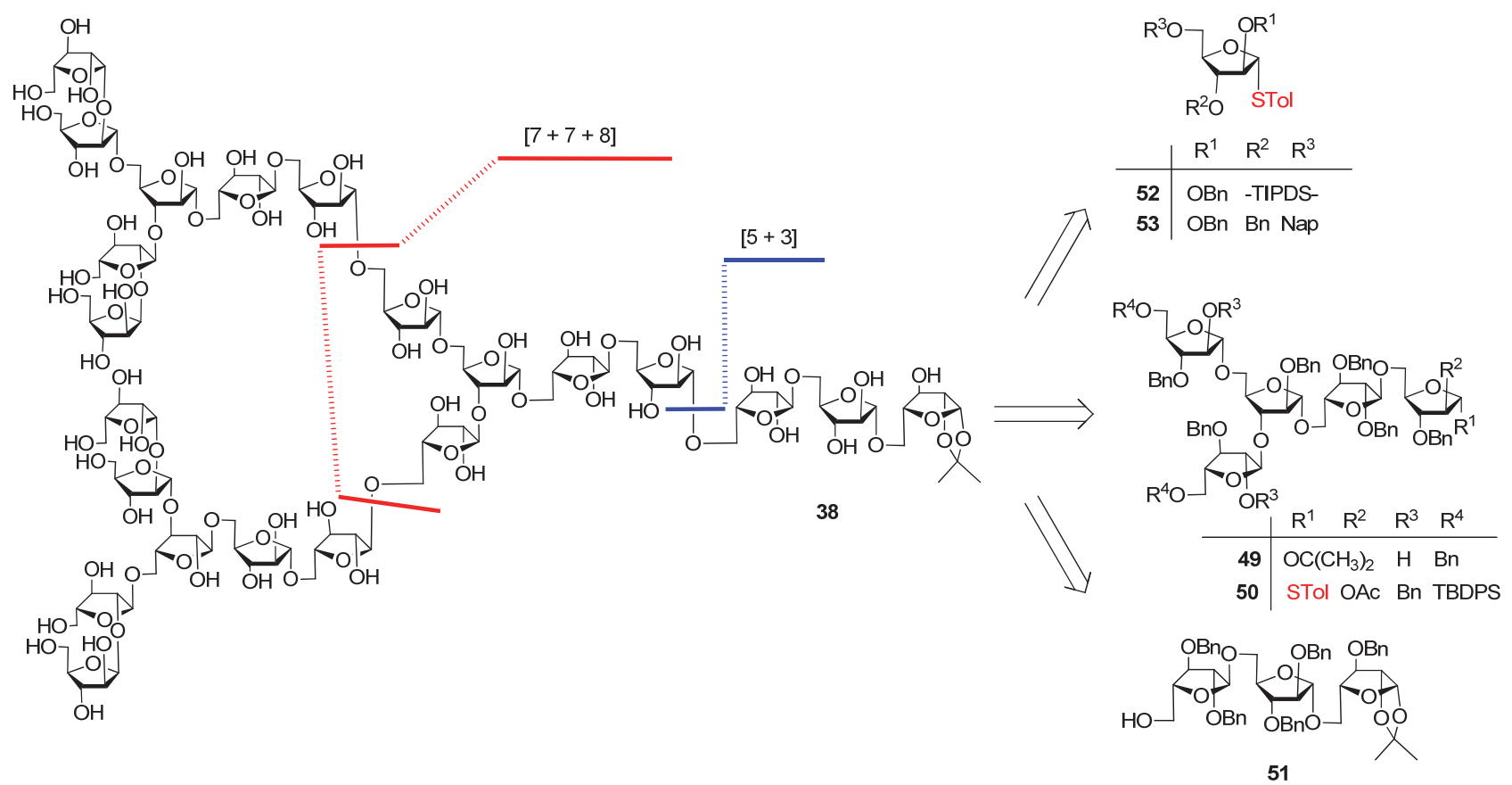

图 17 Ito 小组化学合成结核分枝杆菌细胞壁多糖 38

Figure 17 Ito's chemical synthesis of polysaccharide $\mathbf{3 8}$ of Mycobacterium tuberculosis cell wall

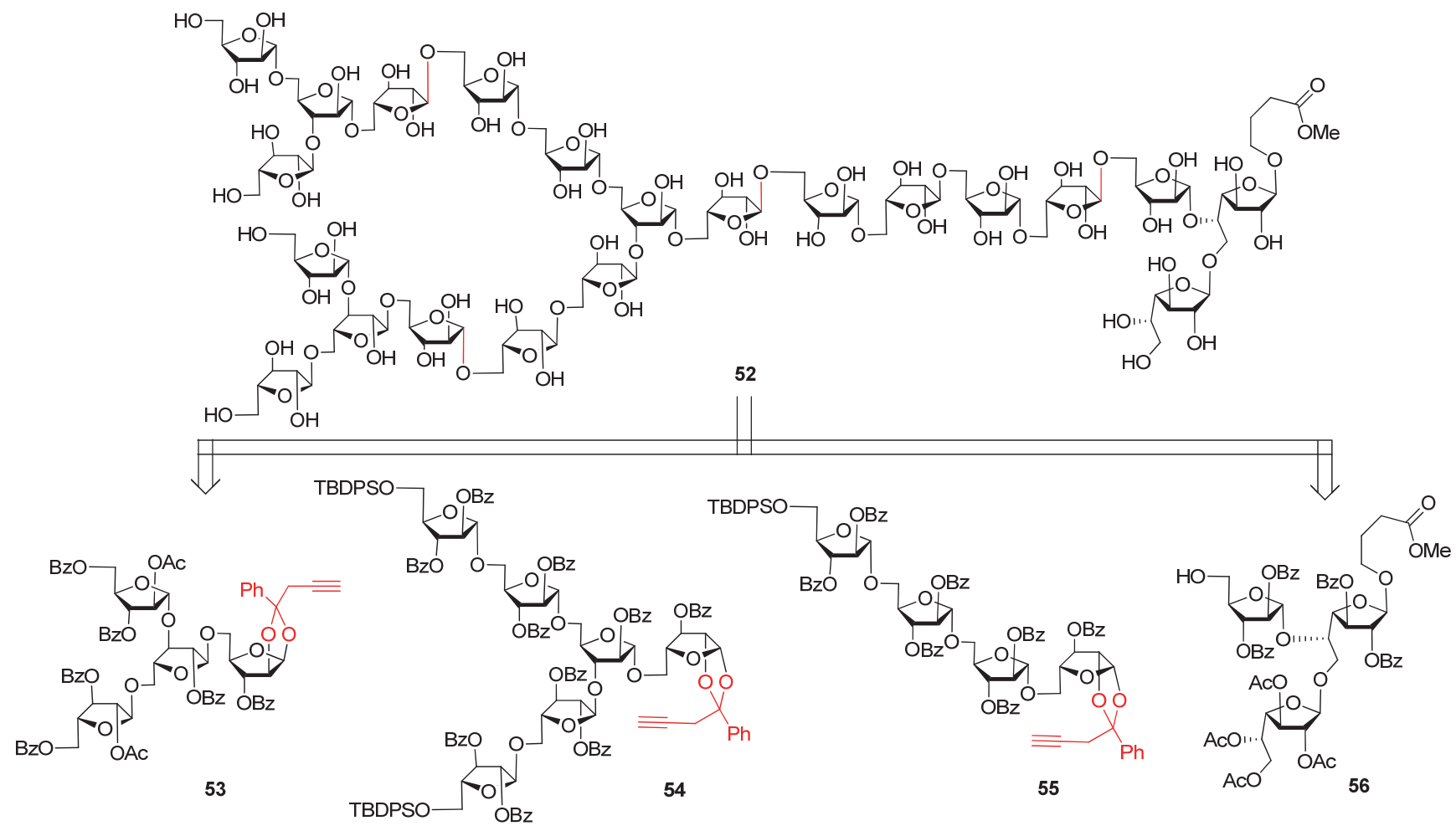

图 18 Hotha 小组化学合成结核分枝杆菌细胞壁多糖 $\mathbf{5 2}$

Figure 18 Hotha's chemical synthesis of polysaccharide 52 of Mycobacterium tuberculosis cell wall

所有的臭糖链 $(62,64,68,70$ 和 74) 均可由适当保护的单 糖硫苷经预活化一釜糖基化反应得到. 值得一提的是, 在最后使用 $\mathrm{Araf}_{31}$ 供体 $\mathbf{5 8}$ 和 $\mathrm{Gal}_{30}$ 受体 $\mathbf{5 9}$ 的双糖基化 反应中, 经过大量的反应条件篮选, 最后发现只有在我
们小组发展的 $\mathrm{BSM} / \mathrm{Tf}_{2} \mathrm{O}$ 活化条件下, 才可以高收率地 完成三条多糖链的定点偶联. 最后经整体去保护, 便完 成了多糖 Arabinogalactan (57)的全合成. 


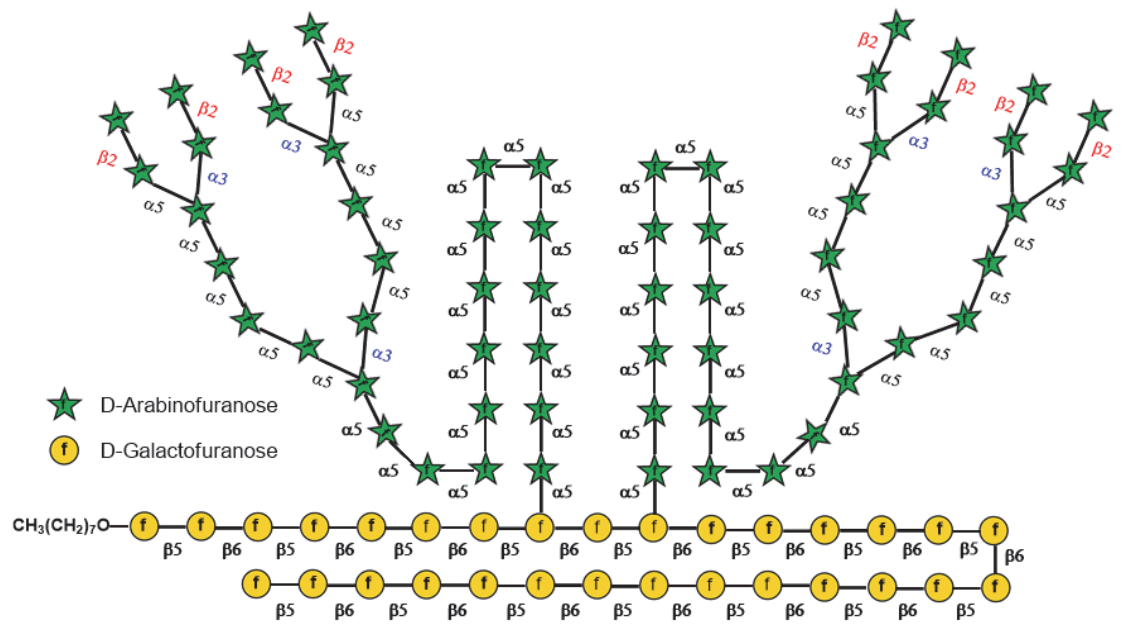

图 19 结核分枝杆菌细胞壁多糖 Arabinogalactan (57)的化学结构

Figure 19 Chemical structure of arabinogalactan polysaccharide (57) of Mycobacterium tuberculosis cell wall

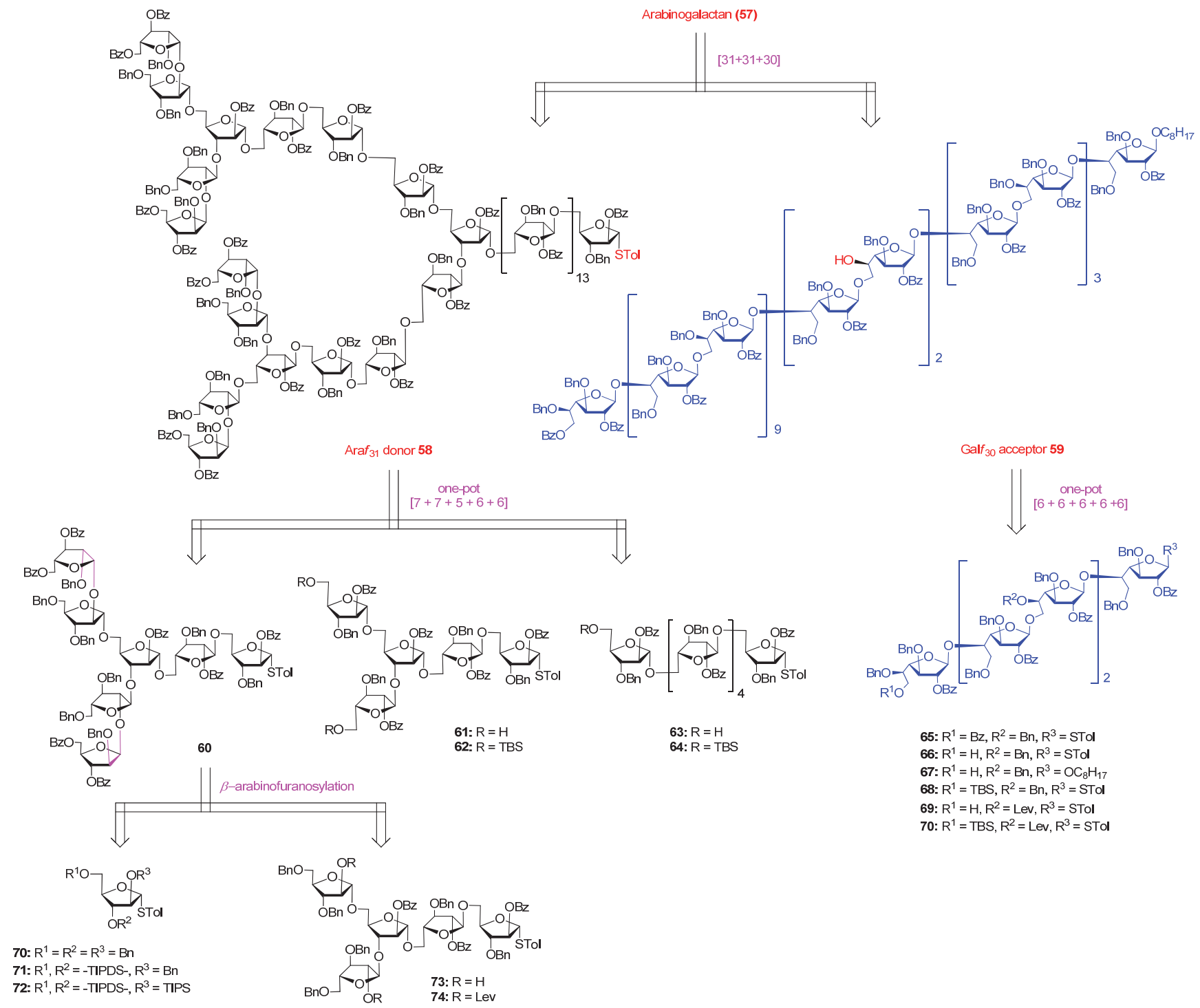

图 20 结核分枝杆菌细胞壁多糖 Arabinogalactan (57)的化学合成

Figure 20 Chemical synthesis of arabinogalactan polysaccharide (57) of Mycobacterium tuberculosis cell wall 
结核分枝杆菌细胞壁中其它重要多糖, 如主要的抗 原性成分脂阿拉伯甘露聚糖(Lipoarabinomannan, LAM) 也有多个片段合成的报道. 代表性的例子如 Fraser-Reid 小组 ${ }^{[97]} 2006$ 年完成的全保护二十八糖和近期 Hotha 小 组 ${ }^{[98]}$ 报道的阿拉伯甘露二十一糖的合成等.

\section{4 其它类型多糖的化学合成}

2013 年, Seeberger 小组 ${ }^{[99}$ 报道了他们使用自动化 固相合成在多糖合成领域中的重要进展. 如图 21 所示, 他们以 6-位 Fmoc 保护, 其它位点 Bz 保护的甘露糖磷酸 酯 75 为糖基供体, 使用带有紫外下可断裂连接臂 (Linker)的树脂 76 作固相载体, 首先经固相合成得到 29 糖(每步糖基化偶联后使用 $\mathrm{Pyr} / \mathrm{Ac}_{2} \mathrm{O}$ 封闭未反应完全的 受体, 再脱除 Fmoc 以进行下一步糖基化), 最后一步糖 基化使用 6-位带有一个功能基团的糖基供体 77, 固相 合成完成后, 将糖链从树枝上切下来, 再脱除 Fmoc 得 到 78. 由于固相合成中有一部分受体不会完全反应, 因 此从树脂上切下来的是由产物和片段缺失序列组成的 混合物. 为了将 30-mer 产物从混合物中分出, 作者发展 了一种 “捕获-释放” 的方法, 即使用丁二酰亚胺活化的 磁珠 79 作为 “捕获” 试剂, 只有完整的 30-mer 能与磁 珠反应，再将片段缺失序列通过简单的洗涤除去，最后 在 $\mathrm{MeONa} / \mathrm{MeOH}$ 条件下将糖链从磁珠上 “释放” 下来, 同时脱除 $\mathrm{Bz}$, 即得到结构均一的甘露糖三十聚体 $\mathbf{8 1}$.
另外, 释放下来的磁珠 82 可以循环使用.

2017 年, Seeberger 小组 ${ }^{[100]}$ 报道了他们使用自动化 固相合成更长 $\alpha-1,6$ 甘露聚糖的工作. 如图 22 所示, 作 者放弃了上一个工作中所使用的糖基磷酸酯，而换用更 稳定的乙硫苷 83 作供体, 而且在甘露糖 3,4 位使用 Bn 保护以提高供体反应活性. 作者发现随着糖链的不断延 长, n-1 的片段缺失序列越来越多, 而且与完整长度的糖 链在 HPLC 上的分离度也越来越差. 为了解决这个问题, 他们在最后 5 步糖基化时使用了大大过量的糖基供体 $(2$ $\times 6.4$ equiv.), 最终耗时 $250 \mathrm{~h}$, 经过 102 步反应得到部 分保护的 50-mer 84 (5\%总收率，平均每步 97\%). 整体 去保护即完成甘露糖五十聚体 85 合成.

最近, Seeberger 小组 ${ }^{[101]}$ 对自动化固相合成技术中 的 “加帽(Capping)” 反应进行了改进. 他们发现使用 $\mathrm{MsOH} / \mathrm{Ac}_{2} \mathrm{O} / \mathrm{CH}_{2} \mathrm{Cl}_{2}$ 条件代替原先的 $\mathrm{Pyr} / \mathrm{Ac}_{2} \mathrm{O}$ 条件, 可 以显著提高加帽反应的效率，缩短合成时间和提高总收 率. 作者使用改进的自动化固相合成技术重新合成多糖 85 时，总耗时可以缩短至 4 天，并且总收率提高至 $22 \%$.

\section{5 总结与展望}

近几十年来，聚糖的化学合成取得了惊人的进步. 许多性质优良的糖基供体、高效新颖的活化方式以及以 液相一釜合成和自动固相合成为代表的聚糖链组装新

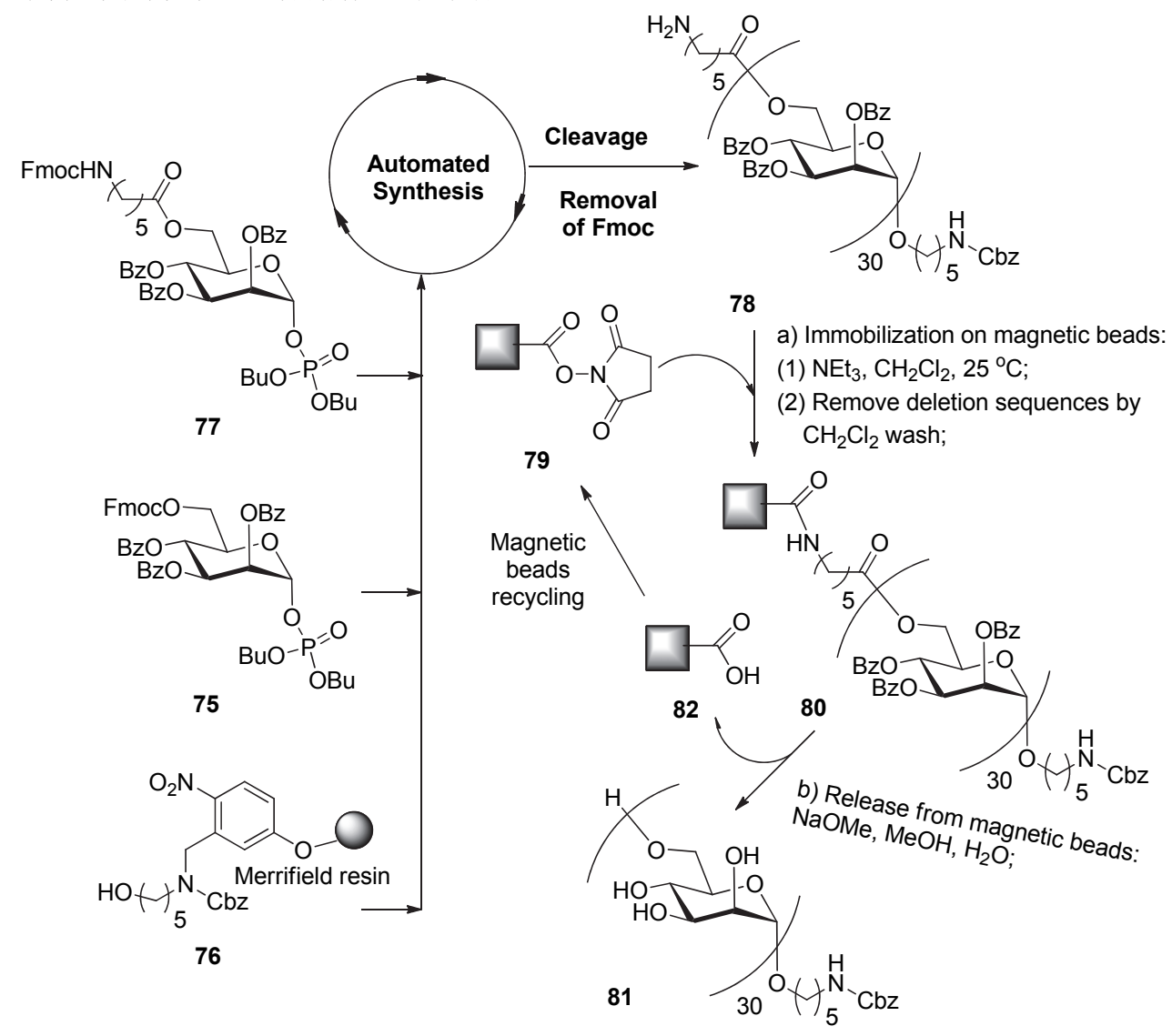

图 21 Seeberger 小组用自动化固相合成技术合成甘露聚糖 30-mer 81

Figure 21 Seeberger's automated solid phase synthesis of a 30-mer mannoside 81 


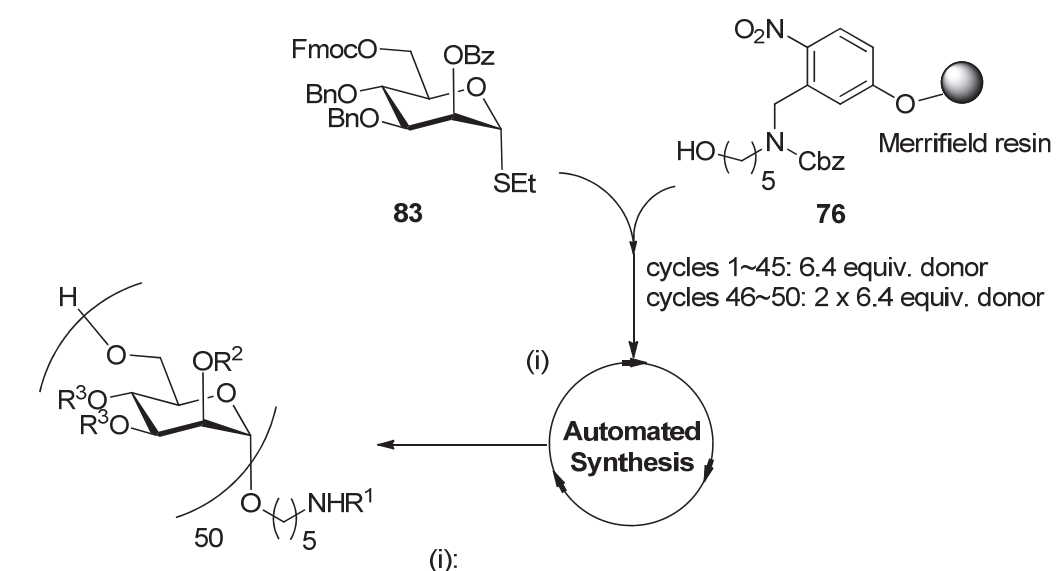

(i):

$$
\begin{aligned}
\text { 84: } R^{1}=C b z, R^{2}=B z, \\
R^{3}=B n \\
\text { 85: } R^{1}=R^{2}=R^{3}=H
\end{aligned}
$$

a) $\mathrm{NaOMe} / \mathrm{MeOH}$, r.t.;

b) $\mathrm{Pd} / \mathrm{C}, \mathrm{H}_{2}$

MeOH:THF:AcOH (9/1/0.1). a) glycosylation: 83 , NIS, $\mathrm{TfOH},-40^{\circ} \mathrm{C}$ to $-20^{\circ} \mathrm{C}$, then $20 \mathrm{~min}, \mathrm{CH}_{2} \mathrm{Cl}_{2}$, dioxane;

b) capping: pyridine, $\mathrm{Ac}_{2} \mathrm{O}$, r.t.;

c) Fmoc deprotection: $\mathrm{Et}_{3} \mathrm{~N}$, DMF, r.t., 5 min, repeated twice;

d) acid wash: TMSOTf, $\mathrm{CH}_{2} \mathrm{Cl}_{2},-20^{\circ} \mathrm{C}$;

e) photocleavage: $h v, \mathrm{CH}_{2} \mathrm{Cl}_{2}$, r.t.

图 22 Seeberger 小组用自动化固相合成技术合成甘露聚糖 50-mer 85

Figure 22 Seeberger's automated solid phase synthesis of a 50-mer mannoside 85

策略的发展和不断成熟, 使得糖化学家们的武器库变得 越来越充实, 很多曾经无法通过化学方法得到的聚糖结 构现在都能合成甚至大量制备.

然而, 和核酸或多肽相比, 聚糖化学合成的技术水 平仍然有待提高. 糖合成化学中还有很多难题没有得到 有效解决, 例如: 糖基化反应的详细机理还有待进一步 探索; 某些糖苷键特别是 1,2-顺式糖苷键选择性构建方 法还比较有限; 糖基化方法或聚糖链组装策略的普适性 还有待提高. 正因为如此, 聚糖的化学合成通常需要精 心的设计和反复的尝试, 迄今为止还是非常依赖具备专 门知识和技能的实验室.

对于自然界广泛存在的多聚糖, 其化学合成、功能 阐释和构效关系等方面的研究还处在起步阶段, 这片处 女地有待糖科学家们去继续探索, 更多的重要进展和有 趣发现值得人们期待.

\section{作者简介}

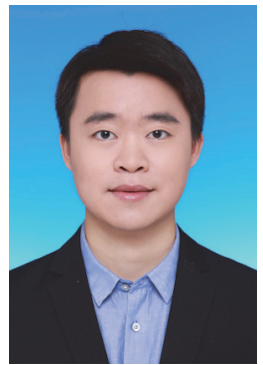

吴勇, 博士, 1990 年出生于四川资阳, 2013 年在四川大学 华西药学院获得学士学位, 随后加入北京大学天然药物及仿 生药物国家重点实验室叶新山教授课题组, 并于 2018 年获得
博士学位. 博士阶段主要研究具有重要生物活性复杂多糖的 化学合成.

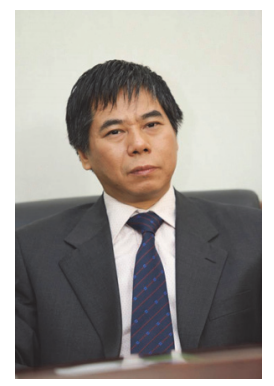

叶新山, 博士, 教授, 分别于 1985 和 1988 年在武汉大学 获得学士和硕士学位, 1996 年在香港中文大学获得博士学位, 之后在美国斯克利普斯研究所从事博士后研究. 2000 年回国 后加入北京大学药学院, 主要研究兴趣包括新的聚糖化学合 成方法和策略的发展、生物活性聚糖(包括肿瘤相关糖抗原) 的合成和活性评价以及基于糖类的新药的发现.

\section{References}

[1] Varki, A. Glycobiology 1993, 3, 97.

[2] Krasnova, L.; Wong, C.-H. Annu. Rev. Biochem. 2016, 85, 599.

[3] Boltje, T. J.; Buskas, T.; Boons, G.-J. Nat. Chem. 2009, 1, 611.

[4] Seeberger, P. H.; Werz, D. B. Nature 2007, 446, 1046.

[5] (a) Gao, Y.; Cao, Z.; Han, Z.; Zhang, Q.; Hu, J.; Guo, R.; He, X.; Ding, F.; You, Q.; Zhang, Y. Chin. J. Org. Chem. 2019, 39, 390. (高 阳光, 曹周, 韩忠享, 张强, 胡杰, 郭锐, 贺贤然, 丁菲, 尤庆亮, 张勇民，有机化学, 2019, 39, 390); (b) Guo, Q.; Wang, X.; Huang, C.; Zhang, P.; Li, Y.; Chen, B. Chin. J. Org. Chem. 2018, 38, 940 (邱果, 王新承, 黄崇品, 张璞, 李英霞, 陈标华, 有机化学, 2018, 38, 940); (c) Yuan, W.; Huang, Y.; Wu, C.; Liu, X.; Xia, Y.; Wang, H. Chin. J. Chem. 2017, 35, 1739; (d) Guo, B.; Ye, L.; Tang, G.; Zhang, L.; Yue, B.; Tsang, S. C. E.; He, H. Chin. J. Chem. 2017, 35, 1529; (e) Mao, R.; Sun, L.; Wang, Y.-S.; Zhou, M.-M.; Xiong, D.-C.; Li, Q.; Ye, X.-S. Chin. Chem. Lett. 2018, 29, 61; (f) Tang, S.; Xiong, D.-C.; Jiang, S.; Ye, X.-S. Org. Lett. 2016, 18, 568.

[6] Bertozzi, C. R.; Kiessling, L. L. Science 2001, 291, 2357. 
[7] Gabius, H.-J. The Sugar Code: Fundamentals of Glycosciences, John Wiley \& Sons, New Jersey, 2011.

[8] Tanaka, H.; Kawai, T.; Adachi, Y.; Hanashima, S.; Yamaguchi, Y.; Ohno, N.; Takahashi, T. Bioorg. Med. Chem. 2012, 20, 3898.

[9] Petitou, M.; Duchaussoy, P.; Driguez, P.-A.; Hérault, J.-P.; Lormeau, J.-C.; Herbert, J.-M. Bioorg. Med. Chem. Lett. 1999, 9, 1155.

[10] Wang, L.; Feng, S.; An, L.; Gu, G.; Guo, Z. J. Org. Chem. 2015, 80, 10060 .

[11] (a) Zhu, X.; Schmidt, R. R. Angew. Chem. Int. Ed. 2009, 48, 1900; (b) Hsu, C.-H.; Hung, S.-C.; Wu, C.-Y.; Wong, C.-H. Angew. Chem. Int. Ed. 2011, 50, 11872; (c) Seeberger, P. H. Acc. Chem. Res. 2015, 48, 1450; (d) Kulkarni, S. S.; Wang, C.-C.; Sabbavarapu, N. M.; Podilapu, A. R.; Liao, P.-H.; Hung, S.-C. Chem. Rev. 2018, 118, 8025.

[12] Michael, A. Am. Chem. J. 1879, 1, 305.

[13] Toshima, K.; Tatsuta, K. Chem. Rev. 1993, 93, 1503.

[14] (a) Garcia, B. A.; Poole, J. L.; Gin, D. Y. J. Am. Chem. Soc. 1997, 119, 7597; (b) Garcia, B. A.; Gin, D. Y. J. Am. Chem. Soc. 2000, 122,4269 .

[15] (a) Schmidt, R. R.; Michel, J. Angew. Chem. Int. Ed. 1980, 19, 731; (b) Schmidt, R. R. Angew. Chem. Int. Ed. 1986, 25, 212

[16] (a) Codée, J. D. C.; Litjens, R. E. J. N.; van den Bos, L. J.; Overkleeft, H. S.; van der Marel, G. A. Chem. Soc. Rev. 2005, 34, 769; (b) Lian, G.; Zhang, X.; Yu, B. Carbohydr. Res. 2015, 403, 13.

[17] Mootoo, D. R.; Konradsson, P.; Udodong, U.; Fraser-Reid, B. J. Am. Chem. Soc. 1988, 110, 5583.

[18] Danishefsky, S. J.; Bilodeau, M. T. Angew. Chem. Int. Ed. 1996, 35, 1380.

[19] Plante, O. J.; Palmacci, E. R.; Andrade, R. B.; Seeberger, P. H. J. Am. Chem. Soc.2001, 123, 9545.

[20] Yu, B. Acc. Chem. Res. 2018, 51, 507.

[21] Koenigs, W.; Knorr, E. Ber. Dtsch. Chem. Ges. 1901, 34, 957.

[22] Zemplén, G.; Gerecs, A. Ber. Dtsch. Chem. Ges. 1930, 63, 2720.

[23] Helferich, B.; Wedemeyer, K. F. Justus Liebigs Ann. Chem. 1949, $563,139$.

[24] Igarashi, K.; Irisawa, J.; Honma, T. Carbohydr. Res. 1975, 39, 213.

[25] Kronzer, F. J.; Schuerch, C. Carbohydr. Res. 1973, 27, 379.

[26] Wulff, G.; Röhle, G.; Krüger, W. Chem. Ber. 1972, 105, 1097.

[27] Yamada, H.; Hayashi, T. Carbohydr. Res. 2002, 337, 581.

[28] Bernstein, S.; Conrow, R. B. J. Org. Chem. 1971, 36, 863

[29] Nishizawa, M.; Garcia, D. M.; Shin, T.; Yamada, H. Chem. Pharm. Bull. 1993, 41, 784 .

[30] Mukaiyama, T.; Murai, Y.; Shoda, S. Chem. Lett. 1981, 10, 431.

[31] Mukaiyama, T.; Hashimoto, Y.; Shoda, S. Chem. Lett. 1983, 12, 935.

[32] Matsumoto, T.; Maeta, H.; Suzuki, K. Tetrahedron Lett. 1988, 29, 3567.

[33] Hashimoto, S.; Hayashi, M.; Noyori, R. Tetrahedron Lett. 1984, 25, 1379.

[34] Mukaiyama, T.; Jona, H.; Takeuchi, K. Chem. Lett. 2000, 29, 696.

[35] Zhu, X.; Schmidt, R. R. Angew. Chem., Int. Ed. 2009, 48, 1900.

[36] El-Badry, M. H.; Gervay-Hague, J. Tetrahedron Lett. 2005, 46, 6727.

[37] (a) Lam, S. N.; Gervay-Hague, J. Carbohydr. Res. 2002, 337, 1953; (b) Lam, S. N.; Gervay-Hague, J. Org. Lett. 2002, 4, 2039; (c) Lam, S. N.; Gervay-Hague, J. J. Org. Chem. 2005, 70, 2387.

[38] Sun, L.; Wu, X.; Xiong, D.-C.; Ye, X.-S. Angew. Chem. Int. Ed. 2016, 55, 8041 .

[39] Park, Y.; Harper, K. C.; Kuhl, N.; Kwan, E. E.; Liu, R. Y.; Jacobsen, E. N. Science 2017, 355, 162.

[40] Schmidt, R. R.; Toepfer, A. Tetrahedron Lett. 1991, 32, 3353.

[41] Yu, B.; Tao, H. Tetrahedron Lett. 2001, 42, 2405.

[42] Ferrier, R. J.; Hay, R. W.; Vethaviyasar, N. Carbohydr. Res. 1973, $27,55$.

[43] Veeneman, G. H.; Van Leeuwen, S. H.; Van Boom, J. H. Tetrahedron Lett. 1990, 31, 1331.

[44] Konradsson, P.; Udodong, U. E.; Fraser-Reid, B. Tetrahedron Lett. 1990, 31, 4313.

[45] Andersson, F.; Fúgedi, P.; Garegg, P. J.; Nashed, M. Tetrahedron Lett. 1986, 27, 3919.

[46] Martichonok, V.; Whitesides, G. M. J. Org. Chem. 1996, 61, 1702.

[47] Crich, D.; Smith, M. J. Am. Chem. Soc. 2001, 123, 9015.

[48] Codée, J. D. C.; Litjens, R. E. J. N.; den Heeten, R.; Overkleeft, H. S.; van Boom, J. H.; van der Marel, G. A. Org. Lett. 2003, 5, 1519.

[49] Wang, C.; Wang, H.; Huang, X.; Zhang, L.-H.; Ye, X.-S. Synlett 2006, 2846.
[50] Marra, A.; Mallet, J. M.; Amatore, C.; Sinaÿ, P. Synlett 1990, 572.

[51] (a) Mitsudo, K.; Kawaguchi, T.; Miyahara, S.; Matsuda, W.; Kuroboshi, M.; Tanaka, H. Org. Lett. 2005, 7, 4649; (b) Nokami, T.; Shibuya, A.; Tsuyama, H.; Suga, S.; Bowers, A. A.; Crich, D.; Yoshida, J. I. J. Am. Chem. Soc. 2007, 129, 10922.

[52] (a) Nakanishi, M.; Takahashi, D.; Toshima, K. Org. Biomol. Chem. 2013, 11, 5079; (b) Wever, W. J.; Cinelli, M. A.; Bowers, A. A. Org. Lett. 2012, 15, 30; (c) Mao, R.-Z.; Guo, F.; Xiong, D.-C.; Li, Q.; Duan, J.; Ye, X.-S. Org. Lett. 2015, 17, 5606; (d) Mao, R.-Z.; Xiong, D.-C.; Guo, F.; Li, Q.; Duan, J.; Ye, X.-S. Org. Chem. Front. 2016 3, 737; (e) Spell, M. L.; Deveaux, K.; Bresnahan, C. G.; Bernard, B. L.; Sheffield, W.; Kumar, R.; Ragains, J. R. Angew. Chem. Int. Ed. 2016, 55, 6515; (f) Yu, Y.; Xiong, D.-C.; Mao, R.-Z.; Ye, X.-S. J. Org. Chem. 2016, 81, 7134. (g) Wang, H.; Wu, P.; Zhao, X.; Zeng, J.; Wan, Q. Acta Chim. Sinica 2019, 77, 231. (王浩, 吴品儒, 赵祥, 曾静, 万谦, 化学学报, 2019, 77, 231.); (h) Ye, H.; Xiao, C.; Lu, L. Chin. J. Org. Chem. 2018, 38, 1897. (叶辉, 肖聪, 陆良秋, 有机化 学, 2018, 38, 1897.)

[53] Goswami, M.; Ellern, A.; Pohl, N. L. B. Angew. Chem. Int. Ed. 2013, 52, 8441.

[54] Yamada, H.; Harada, T.; Miyazaki, H.; Takahashi, T. Tetrahedron Lett. 1994, 35, 3979.

[55] Zhang, Z.; Ollmann, I. R.; Ye, X.-S.; Wischnat, R.; Baasov, T.; Wong, C.-H. J. Am. Chem. Soc. 1999, 121, 734.

[56] Huang, X.; Huang, L.; Wang, H.; Ye, X.-S. Angew. Chem Int. Ed. 2004, 43, 5221.

[57] Plante, O. J.; Palmacci, E. R.; Seeberger, P. H. Science 2001, 291, 1523.

[58] Tanaka, H.; Adachi, M.; Tsukamoto, H.; Ikeda, T.; Yamada, H.; Takahashi, T. Org. Lett. 2002, 4, 4213.

[59] Yu, B.; Yu, H.; Hui, Y.; Han, X. Tetrahedron Lett. 1999, 40, 8591.

[60] Wang, P.; Lee, H.; Fukuda, M.; Seeberger, P. H. Chem. Commun. 2007, 1963.

[61] Vohra, Y.; Buskas, T.; Boons, G.-J. J. Org. Chem. 2009, 74, 6064

[62] (a) Hsu, C.-H.; Chu, K. C.; Lin, Y. S.; Han, J. L.; Peng, Y. S.; Ren, C. T.; Wong, C.-H. Chem. Eur. J. 2010, 16, 1754; (b) Tanaka, H.; Tateno, Y.; Nishiura, Y.; Takahashi, T. Org. Lett. 2008, 10, 5597; (c) Tanaka, H.; Adachi, M.; Takahashi, T. Chem. Eur. J. 2005, 11, 849.

[63] (a) Dinkelaar, J.; Gold, H.; Overkleeft, H. S.; Codée, J. D.; van der Marel, G. A. J. Org. Chem. 2009, 74, 4208; (b) Hu, Y. P.; Lin, S. Y.; Huang, C. Y.; Zulueta, M. M. L.; Liu, J. Y.; Chang, W.; Hung, S.-C. Nat. Chem. 2011, 3, 557.

[64] Sarkar, S.; Dutta, S.; Das, G.; Sen, A. K. Tetrahedron 2011, 67, 4118.

[65] Burkhart, F.; Zhang, Z.; Wacowich-Sgarbi, S.; Wong, C.-H. Angew. Chem. Int. Ed. 2001, 40, 1274.

[66] Tsai, B. L.; Han, J. L.; Ren, C. T.; Wu, C.-Y.; Wong, C.-H. Tetrahedron Lett. 2011, 52, 2132.

[67] Mong, K. K. T.; Wong, C.-H. Angew. Chem. Int. Ed. 2002, 41, 4087.

[68] Lee, J. C.; Wu, C.-Y.; Apon, J. V.; Siuzdak, G.; Wong, C.-H. Angew. Chem. Int. Ed. 2006, 45, 2753.

[69] Mong, T. K. K.; Lee, H. K.; Durón, S. G.; Wong, C.-H. PNAS 2003, 100, 797.

[70] Polat, T.; Wong, C.-H. J. Am. Chem. Soc. 2007, 129, 12795.

[71] Hsu, Y.; Lu, X. A.; Zulueta, M. M. L.; Tsai, C. M.; Lin, K. I.; Hung, S.-C.; Wong, C.-H. J. Am. Chem. Soc. 2012, 134, 4549.

[72] Wang, Z.; Zhou, L.; El-Boubbou, K.; Ye, X.-S.; Huang, X. J. Org. Chem. 2007, 72, 6409.

[73] Li, Q.; Guo, Z. Org. Lett. 2017, 19, 6558.

[74] Huang, L.; Huang, X. Chem. Eur. J. 2007, 13, 529.

[75] Miermont, A.; Zeng, Y.; Jing, Y.; Ye, X.-S.; Huang, X. J. Org. Chem. 2007, 72, 8958.

[76] Wang, Z.; Xu, Y.; Yang, B.; Tiruchinapally, G.; Sun, B.; Liu, R.; Huang, X. Chem. Eur. J. 2010, 16, 8365.

[77] Sun, B.; Srinivasan, B.; Huang, X. Chem. Eur. J. 2008, 14, 7072.

[78] Wang, Y.-S.; Wu, Y.; Xiong, D.-C.; Ye, X.-S. Chin. J. Chem. 2019, 37,42 .

[79] (a) Gao, J.; Guo, Z. J. Org. Chem. 2013, 78, 12717; (b) Gao, J.; Liao, G.; Wang, L.; Guo, Z. Org. Lett. 2014, 16, 988; (c) Gao, J.; Guo, Z. Org. Lett. 2016, 18, 5552. (d) Wang, D.; Xiong, D.-C.; Ye, X.-S. Chin. Chem. Lett. 2018, 29, 1340; (e) Wu, Y.; Xiong, D.-C.; Chen, S.-C.; Wang, Y.-S.; Ye, X.-S. Nat. Commun. 2017, 8, 14851.

[80] Werz, D. B.; Castagner, B.; Seeberger, P. H. J. Am. Chem. Soc. 2007, 129, 2770.

[81] Routenberg, L. K.; Seeberger, P. H. Angew. Chem. Int. Ed. 2004, 43, 602. 
[82] Ratner, D. M.; Swanson, E. R.; Seeberger, P. H. Org. Lett. 2003, 5, 4717.

[83] Codée, J. D. C.; Kröck, L.; Castagner, B.; Seeberger, P. H. Chem. Eur. J. 2008, 14, 3987.

[84] (a) Walvoort, M. T. C.; Volbeda, A. G.; Reintjens, N. R. M.; van den Elst, H.; Plante, O. J.; Overkleeft, H. S.; Codée, J. D. Org. Lett. 2012, 14, 3776; (b) Hahm, H. S.; Broecker, F.; Kawasaki, F.; Mietzsch, M.; Heilbronn, R.; Fukuda, M.; Seeberger, P. H. Chem 2017, 2,114 .

[85] Hewitt, M. C.; Snyder, D. A.; Seeberger, P. H. J. Am. Chem. Soc. 2002, 124, 13434.

[86] Matsuzaki, Y.; Ito, Y.; Nakahara, Y.; Ogawa, T. Tetrahedron Lett. 1993, 34, 1061.

[87] Hansen, S. U.; Miller, G. J.; Cliff, M. J.; Jayson, G. C.; Gardiner, J. M. Chem. Sci. 2015, 6, 6158.

[88] Li, A.; Kong, F. Bioorg. Med. Chem. 2005, 13, 839.

[89] Pozsgay, V. Angew. Chem. Int. Ed. 1998, 37, 138.

[90] Pozsgay, V.; Chu, C.; Pannell, L.; Wolfe, J.; Robbins, J. B.; Schneerson, R. PNAS 1999, 96, 5194.
[91] Pozsgay, V. Tetrahedron: Asymmetry 2000, 11, 151.

[92] Joe, M.; Bai, Y.; Nacario, R. C.; Lowary, T. L. J. Am. Chem. Soc. 2007, 129, 9885.

[93] Ishiwata, A.; Ito, Y. J. Am. Chem. Soc. 2011, 133, 2275.

[94] Thadke, S. A.; Mishra, B.; Islam, M.; Pasari, S.; Manmode, S.; Rao, B. V.; Hotha, S. Nat. Commun. 2017, 8, 14019.

[95] Pasari, S.; Manmode, S.; Walke, G.; Hotha, S. Chem. Eur. J. 2018, $24,1128$.

[96] Mishra, B.; Neralkar, M.; Hotha, S. Angew. Chem. Int. Ed. 2016, 55, 7786.

[97] Fraser-Reid, B.; Lu, J.; Jayaprakash, K. N.; Lopez, J. C. Tetrahedron: Asymmetry 2006, 17, 2449.

[98] Islam, M.; Shinde, G. P.; Hotha, S. Chem. Sci. 2017, 8, 2033.

[99] Calin, O.; Eller, S.; Seeberger, P. H. Angew. Chem. Int. Ed. 2013, 52, 5862.

[100] Naresh, K.; Schumacher, F.; Hahm, H. S.; Seeberger, P. H. Chem. Commun. 2017, 53, 9085.

[101] Yu, Y.; Kononov, A.; Delbianco, M.; Seeberger, P. H. Chem. Eur. J. 2018, 24, 6075 .

(Cheng, B.) 